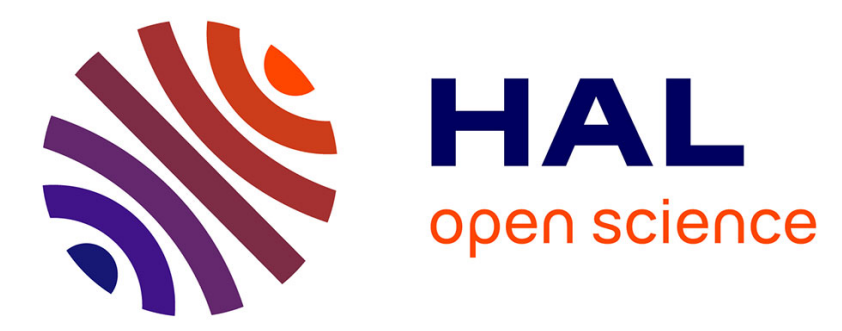

\title{
A method to couple HEM and HRM two-phase flow models
}

\author{
Annalisa Ambroso, Jean-Marc Hérard, Olivier Hurisse
}

\section{To cite this version:}

Annalisa Ambroso, Jean-Marc Hérard, Olivier Hurisse. A method to couple HEM and HRM twophase flow models. Computers and Fluids, 2009, 38, pp.738-756. 10.1016/j.compfluid.2008.04.016 . hal-01265379

\section{HAL Id: hal-01265379 \\ https://hal.science/hal-01265379}

Submitted on 1 Feb 2016

HAL is a multi-disciplinary open access archive for the deposit and dissemination of scientific research documents, whether they are published or not. The documents may come from teaching and research institutions in France or abroad, or from public or private research centers.
L'archive ouverte pluridisciplinaire HAL, est destinée au dépôt et à la diffusion de documents scientifiques de niveau recherche, publiés ou non, émanant des établissements d'enseignement et de recherche français ou étrangers, des laboratoires publics ou privés. 


\title{
A METHOD TO COUPLE HEM AND HRM TWO-PHASE FLOW MODELS
}

\author{
Annalisa Ambroso ${ }^{3}$, Jean-Marc Hérard ${ }^{1 *}$, Olivier Hurisse ${ }^{1,2 \dagger}$ \\ ${ }^{1}$ Électricité de France, Division Recherche et Développement, \\ Département Mécanique des Fluides, Energies et Environnement, \\ 6 quai Watier, 78401 Chatou cedex, FRANCE \\ jean-marc.herard@edf.fr, olivier.hurisse@edf.fr \\ ${ }^{2}$ Université de Provence, Centre de Mathématiques et d'Informatique, \\ Laboratoire d'Analyse, Topologie et Probabilités - UMR CNRS 6632, \\ 39 rue Joliot Curie, 13453 Marseille cedex 13, FRANCE \\ ${ }^{3}$ Commissariat à l'Energie Atomique, \\ Centre de Saclay, DEN-DM2S-SFME-LETR , \\ F-91191 Gif-sur-Yvette, France, \\ annalisa.ambroso@cea.fr
}

\begin{abstract}
We present a method for the unsteady coupling of two distinct two-phase flow models (namely the Homogeneous Relaxation Model, and the Homogeneous Equilibrium Model) through a thin interface. The basic approach relies on recent works devoted to the interfacial coupling of CFD models, and thus requires to introduce an interface model. Many numerical test cases enable to investigate the stability of the coupling method.
\end{abstract}

\section{Introduction}

We focus in this paper on the unsteady interfacial coupling of two distinct two-phase flow models that are commonly used in order to simulate water-vapour flows in nuclear power plants. We emphasize that we only deal with a steady coupling interface that separates the two codes. The main objective here is to prescribe meaningful boundary conditions on each side of this coupling interface for both codes associated with HEM and HRM models.

The Homogeneous Relaxation Model (denoted by the acronym HRM) is a four-equation model that is widely used in two-phase flow simulations. Most industrial codes within the nuclear community - for instance THYC (EDF) or FLICA (CEA) - rely on this model. This model requires computing approximations of solutions of two mass balance equations, a total momentum equation and a governing equation for the total energy balance of the mixture. Excluding source terms, this model is under conservative form. The only non-zero source contribution is on the right hand side of the governing equation of the liquid mass fraction.

\footnotetext{
${ }^{*}$ Corresponding author

${ }^{\dagger} \mathrm{PhD}$ student

Copyright (C) 2006 by Electricité de France
} 
This source tends to relax the current liquid mass fraction to the equilibrium mass fraction, which only depends on the mean pressure and the mean density. The underlying time scale is highly variable, and in practice it makes the source term very stiff, which may render the computation of the HRM model rather uneasy. Actually, the Homogeneous Equilibrium Model (acronym HEM) precisely stands for the counterpart of the HRM model when an equilibrium is achieved. It is thus a pure convective set of partial differential equations which govern the motion of the total mass, the global momentum and the total energy of the whole mixture. Both the HEM and the HRM models require defining appropriate equations of state (referred to as the EOS in the following) in order to account for both the "pure vapour" phase, the "pure liquid" phase but also the "mixture" phase. These EOS are usually tabulated ( $\operatorname{see}^{22,30,31}$ ), but we will focus here on simplified analytical EOS. This is essentially motivated by the fact that we do not wish to mix numerical drawbacks due to the use of realistic EOS and those connected with the formulation of the coupling techniques. In other words, we want to be "optimal" in some sense in terms of EOS in order to concentrate on the main drawbacks of the coupling techniques.

In order to introduce the problem of the interfacial coupling of two existing codes, we need to define governing equations:

$$
\partial_{t}(W)+\partial_{n}\left(F_{n, L}(W)\right)=0
$$

for the left code $\left(x_{n}=x \cdot n<0, t>0\right)$, respectively, for the right code $\left(x_{n}>0, t>0\right)$ :

$$
\partial_{t}(W)+\partial_{n}\left(F_{n, R}(W)\right)=0
$$

where $n$ is the unit normal to the plane and steady coupling interface, which is located at $x_{n}=0$. Moreover, we assume that the two systems on each side are hyperbolic and invariant under frame rotation.

Quite recently, some authors have proposed two approaches in order to tackle the unsteady interfacial coupling of CFD models. Roughly speaking, the first approach favours the continuity of the conservative variable $W$, by enforcing $W\left(x_{n}=0^{-}, t\right)=W\left(x_{n}=0^{+}, t\right)$ in a weak sense $\left(\operatorname{see}^{18,20}\right)$. This method has been recently extended to the case of a generic variable $Z(W)\left(\mathrm{see}^{2,4}\right.$ and also $\left.{ }^{3,10,13}\right)$. The second one relies on the basic paper by Greenberg and Leroux. ${ }^{21}$ It consists in introducing a colour function $Y(x, t)$ where $Y(x, t)=1$, if $x_{n}=x \cdot n<0$, and $Y(x, t)=0$ if $x_{n}=x \cdot n>0$. Since the interface is steady, the function $Y$ verifies $\partial_{t}(Y)=0$. Defining $F_{n}(W)=Y F_{n, L}(W)+(1-Y) F_{n, R}(W)$, the fluxes at the steady coupling interface can be computed by solving the Riemann problem associated with:

$$
\partial_{t}(W)+\partial_{n}\left(F_{n}(W)\right)=0,
$$

This method, which introduces the "father model" (3) obviously privileges the conservation law. It has been used in. ${ }^{23,26}$

More recently, a third approach has been proposed $\left({ }^{2,4}\right)$. It combines the second method with the relaxation methods. ${ }^{5,6,11}$ The coupling technique that is used herein makes use of the latter approach. Actually, we want here to take advantage of the fact that the HRM model may be viewed as the "father model" of the HEM model. Another advantage of the third approach is that one may get rid of possible resonance phenomena, as underlined $\mathrm{in}^{2,4}$ for instance. This phenomena may arise when using the second approach if a genuinely non linear field overlaps the steady linearly degenerate field associated with the colour function $Y$. Though it is not clear whether this has drastic consequences, it seems indeed much more reasonable to avoid this problem that is not clearly understood $\left({ }^{17}\right)$.

The paper is organized as follows:

- Sections II and III are devoted to the presentation of both HEM and HRM models, but also on some of their properties (hyperbolicity, entropy inequality, positivity results for sufficiently smooth solutions).

- We then present the coupling method in section IV. Special attention will be paid to the numerical treatment of the coupling interface, which relies on:

(i) an evolution step,

(ii) an instantaneous relaxation step,

(iii) a finite relaxation step in order to account for source terms. 
This section also includes a brief description of the Finite Volume methods that will be applied in order to compute approximations of solutions in non coupled codes.

- Numerical results are displayed in section V. This includes basic test cases involving contact waves, shock waves and rarefaction waves, but also a schematic representation of the flow in a part of the primary coolant circuit in a nuclear power plant.

Throughout the paper, we will use the following notations: $\rho$ stands for the density of the mixture, $\tau=1 / \rho$ is its specific volume and $U$ represents the mean velocity of the mixture. Moreover $P, C, e$, $h=e+P / \rho, E=e+U^{2} / 2$ respectively stand for the pressure, the liquid mass fraction, the internal energy, the enthalpy, and the total volumetric energy of the mixture. The subscripts $v$ and $l$ respectively refer to the vapor and the liquid phases. The over-script $s$ denotes saturated quantities. 


\section{The Homogeneous Relaxation Model}

This four-equation model can be derived from the six-equation two-fluid model. In the following we focus on specific closure laws and we detail some properties connected with these choices.

\section{A. Closure laws}

We consider that the two fluids have the same mean velocity, that is $U_{l}-U_{v}=U_{r}=0$. In order to take into account the mass transfer between the two phases, a source term $\rho \Gamma$ stands on the right hand side in the equation of the mass balance of the liquid phase. Thus the governing equations are:

$$
\left\{\begin{array}{l}
\partial_{t}(\rho C)+\partial_{x}(\rho C U)=\rho \Gamma \\
\partial_{t}(\rho)+\partial_{x}(\rho U)=0 \\
\partial_{t}(\rho U)+\partial_{x}\left(\rho U^{2}+P\right)=0 \\
\partial_{t}\left(\rho E_{H R M}\right)+\partial_{x}\left(U\left(\rho E_{H R M}+P\right)\right)=0
\end{array}\right.
$$

with:

$$
E_{H R M} \stackrel{\text { def }}{=} e_{H R M}(P, \rho, C)+\frac{U^{2}}{2} \text { and } e_{H R M}(P, \rho, C) \stackrel{\text { def }}{=} h_{H R M}(P, \rho, C)-\frac{P}{\rho}
$$

where the function $h_{H R M}(P, \rho, C)$ is the specific enthalpy that must be prescribed by the user. In practice here, we will use the definition (10).

The set of physical relevant states for the system (4) is:

$$
\Omega_{H R M} \stackrel{\text { def }}{=}\{(\rho, U, P, C) / \rho \geq 0, C \in[0,1], P \geq 0\}
$$

In order to close the system, we need to define $h_{H R M}$ and $\Gamma$.

- First, we write the enthalpy function $h_{H R M}$. We choose the thermodynamic closures inspired by the THYC and FLICA codes. ${ }^{22,31}$ They consider the medium as the mixing of two fluids: liquid water and vapour. Moreover, the vapour is assumed to be in a saturation state, this implies that each thermodynamic function relative to this fluid only depends on one variable, say the pressure $P$. The two pure fluids are assumed to obey a usual $\gamma$ closure law.

We note $\gamma_{v}>1$ (respectively $\gamma_{l}>1$ ) the adiabatic constant for the vapour (respectively the liquid), and $e_{v}, h_{v}, \rho_{v}$ and $\tau_{v}$ the internal specific energy, specific enthalpy, density and specific volume for the vapour (respectively $e_{l}, h_{l}, \rho_{l}$ and $\tau_{l}$ the internal specific energy, specific enthalpy, density and specific volume for the liquid).

We recall the following definitions:

$$
e_{p}(\rho, P) \stackrel{\text { def }}{=} \frac{P}{\left(\gamma_{p}-1\right) \rho} \text { and } h_{p}(\rho, P) \stackrel{\text { def }}{=} \delta_{p} P \tau \text { with } \delta_{p} \stackrel{\text { def }}{=} \gamma_{p} /\left(\gamma_{p}-1\right)
$$

for $p=l, v$.

We will use standard values in order to account for liquid and gas respectively : $\gamma_{l}=1.001$ and $\gamma_{v}=1.4$. Moreover, we assume that if the pressure $P$ stays in $\left[P_{M I N}, P_{M A X}\right]$ the saturation curves for the enthalpy and the volumetric fraction can be approached by the following functions:

- Saturated Vapor:

$$
h_{v}^{s}(P) \stackrel{\text { def }}{=} A_{v} P+B_{v} \quad \text { and } \quad \tau_{v}^{s}(P) \stackrel{\text { def }}{=} \frac{h_{v}^{s}(P)}{\delta_{v} P}
$$

- Saturated Liquid

$$
h_{l}^{s}(P) \stackrel{\text { def }}{=} A_{l} P+B_{l} \quad \text { and } \quad \tau_{l}^{s}(P) \stackrel{\text { def }}{=} \frac{h_{l}^{s}(P)}{\delta_{l} P}
$$


Physically relevant saturation curves ensure that: $\tau_{v}^{s}(P)>\tau_{l}^{s}(P)$ and $h_{v}^{s}(P)>h_{l}^{s}(P)$. Typical values of the coefficients $P_{M I N}, P_{M A X}, A_{v}<0, A_{l}, B_{v}$ and $B_{l}$ related to nuclear cooling conditions can be found at the beginning of Section V.

We can now define the total specific enthalpy of the mixture:

$$
h_{H R M}(P, \rho, C) \stackrel{\text { def }}{=} C h_{l}\left(P, \rho_{l}\right)+(1-C) h_{v}^{s}(P),
$$

where

$$
\rho_{l}=\rho C \frac{1}{1-\rho(1-C) \tau_{v}^{s}(P)}=\frac{C}{\tau-(1-C) \tau_{v}^{s}(P)},
$$

This relation (11) is obtained by introducing the two void fractions $\alpha_{l}$ and $\alpha_{v}$ for the liquid and vapour respectively, that are in agreement with $\alpha_{l}+\alpha_{v}=1$. Using the standard definitions of the partial mass for the liquid and the mean density of the mixture:

$$
\alpha_{l} \rho_{l}=\rho C \quad \text { and: } \quad \rho=\alpha_{l} \rho_{l}+\alpha_{v} \rho_{v}
$$

one may eliminate $\alpha_{l, v}$ and inject $\rho_{v}=\rho_{v}^{s}(P)$, in order to obtain $\rho_{l}$ in terms of $P, \rho, C$, that is the above relation (11).

In the following, the HRM model will refer to the set of equations (4), (5), (10).

The enthalpy (10) of the model can be simplified, by using (7), (8).

$$
\rho h_{H R M}(P, \rho, C)=\delta_{l} P+\rho(1-C) h_{v}^{s}(P)\left(1-\frac{\delta_{l}}{\delta_{v}}\right)
$$

This simplified form of EOS will enable us to derive some technical results.

\section{Remark 1:}

With this thermodynamic closure, the domain of validity of the HRM model is:

$$
D_{H R M} \stackrel{\text { def }}{=}\left\{C \in[0,1], P \in\left[P_{M I N}, P_{M A X}\right],(1-C) \tau_{v}^{s}(P) \leq \tau \leq \tau_{v}^{s}(P)\right\}
$$

where we have used (6), (11) and the constraint $\left.\left.\tau_{l} \in\right] 0, \tau_{v}^{s}(P)\right]$ to obtain the relation on the bounds of $\tau$.

- The source term $\Gamma$ in the set of equations (4) allows the exchange of mass between the two phases. It depends on the density $\rho$, the pressure $P$ and the liquid mass fraction $C$. Its form arises from the literature $\left(\mathrm{see}^{22,31}\right)$ :

$$
\Gamma=\frac{C(1-C)}{\tau_{0}} \rho \tau_{v}^{s}(P) \frac{h_{l}^{s}(P)-h_{l}\left(P, \rho_{l}\right)}{h_{v}^{s}(P)-h_{l}^{s}(P)}
$$

where $\tau_{0}$ is a time scale.

For regular solutions, system ((4), (5)) can be written:

$$
\left\{\begin{array}{l}
\partial_{t}(C)+U \partial_{x}(C)=\Gamma \\
\partial_{t}(\rho)+U \partial_{x}(\rho)+\rho \partial_{x}(U)=0 \\
\partial_{t}(U)+U \partial_{x}(U)+\frac{1}{\rho} \partial_{x}(P)=0 \\
\partial_{t}(P)+U \partial_{x}(P)+\hat{\gamma}_{H R M} P \partial_{x}(U)=-\Gamma \frac{\partial_{C}\left(e_{H R M}\right)}{\partial_{P}\left(e_{H R M}\right)}
\end{array}\right.
$$

with:

$$
\hat{\gamma}_{H R M} \stackrel{\text { def }}{=}\left(P \partial_{P}\left(e_{H R M}\right)\right)^{-1}\left(\frac{P}{\rho}-\rho \partial_{\rho}\left(e_{H R M}\right)\right)
$$

We will use this form to highlight some of its properties.

$$
5 \text { of } 40
$$




\section{B. Hyperbolicity of the HRM model}

In order to study the hyperbolicity of the HRM model, we focus on the convective part of (15) complemented with (5). Then the non-conservative equations on $\rho, U, P$ and $C$ are:

$$
\partial_{t}\left(\begin{array}{c}
C \\
\rho \\
U \\
P
\end{array}\right)+\left(\begin{array}{cccc}
U & 0 & 0 & 0 \\
0 & U & \rho & 0 \\
0 & 0 & U & \tau \\
0 & 0 & \hat{\gamma}_{H R M} P & U
\end{array}\right) \partial_{x}\left(\begin{array}{c}
C \\
\rho \\
U \\
P
\end{array}\right)=\left(\begin{array}{c}
0 \\
0 \\
0 \\
0
\end{array}\right)
$$

The following result is classic:

\section{Property 1}

The system (16) is hyperbolic if and only if $\hat{\gamma}_{H R M} P>0$. Its eigenvalues and right eigenvectors are recalled below:

$$
\begin{array}{ll}
\lambda_{1}=U-a_{H R M} ; & r_{1}^{\top}=\left(0, \rho,-a_{H R M}, \rho a_{H R M}^{2}\right) \\
\lambda_{2}=U ; & r_{2}^{\top}=(1,0,0,0) \\
\lambda_{3}=U ; & r_{3}^{\top}=(0,1,0,0) \\
\lambda_{4}=U+a_{H R M} ; & r_{4}^{\top}=\left(0, \rho,+a_{H R M}, \rho a_{H R M}^{2}\right)
\end{array}
$$

where we define the sound velocity $a_{H R M}$ by:

$$
a_{H R M} \stackrel{\text { def }}{=}\left(\hat{\gamma}_{H R M} P / \rho\right)^{1 / 2}
$$

\section{Property 2}

If we assume that :

$$
1<\gamma_{l}<\gamma_{v} \quad \text { and } A_{v}<0
$$

the system (16) is hyperbolic for all $P \in\left[P_{M I N}, P_{M A X}\right], \rho>0, C \in[0,1]$.

We insist that this is not a necessary condition.

Proof

Using the definition (12) of $h_{H R M}$ together with the relation $\rho e_{H R M}=\rho h_{H R M}-P$ we obtain:

$$
\begin{gathered}
\left(\frac{P}{\rho}-\rho \partial_{\rho}\left(e_{H R M}\right)\right)=\frac{\delta_{l} P}{\rho} \\
\partial_{P}\left(e_{H R M}\right)=\tau\left(\delta_{l}-1\right)+(1-C)\left(1-\frac{\delta_{l}}{\delta_{v}}\right) \frac{d}{d P} h_{v}^{s}(P)
\end{gathered}
$$

The term $\frac{\delta_{l} P}{\rho}$ is always positive, and a sufficient condition for $\partial_{P}\left(e_{H R M}\right)$ to be positive is:

$$
\left(1-\frac{\delta_{l}}{\delta_{v}}\right) \frac{d}{d P} h_{v}^{s}(P)>0
$$

Thanks to (8), we have: $\frac{d}{d P} h_{v}^{s}(P)=A_{v}$, and $A_{v}$ is negative. Obviously, $1<\gamma_{l}<\gamma_{v}$ implies: $\delta_{v}<\delta_{l}$. Thus, $\hat{\gamma}_{H R M} P$ is positive, which concludes the proof.

We remark that the numerical values in section $\mathrm{V}$, equation (42), are such that $A_{v}<0$, and that $\gamma_{l, v}$ fulfill the condition above.

\section{Some positivity results for the HRM model}

Remark 2:

We have introduced the source term $\Gamma$ in (14). We can rewrite it in a form which will appear to be more convenient. We set :

$$
G_{2}(P, \rho) \stackrel{\text { def }}{=} \rho \tau_{v}^{s} \delta_{l} P \frac{\tau_{v}^{s}(P)-\tau_{l}^{s}(P)}{h_{v}^{s}(P)-h_{l}^{s}(P)} \quad \text { and } \quad \bar{C} \stackrel{\text { def }}{=} \frac{\tau_{v}^{s}(P)-\tau}{\tau_{v}^{s}(P)-\tau_{l}^{s}(P)}
$$

6 of 40 
Since the saturation functions ensure that both $\tau_{v}^{s}(P)>\tau_{l}^{s}(P)$ and $h_{v}^{s}(P)>h_{l}^{s}(P)$, we know that $G_{2}>0$ if $P \in\left[P_{M I N}, P_{M A X}\right]$, and if $\rho>0$. The source term may thus be rewritten:

$$
\Gamma=(1-C)(\bar{C}-C) \frac{G_{2}(P, \rho)}{\tau_{0}}
$$

The $O D E$

$$
\partial_{t}(C)=\Gamma(P, \rho, C)
$$

has two poles. The pole $C=1$ is repulsive and the pole $C=\bar{C}$ is attractive. The expression of the second pole will make sense for the HEM model. This remark will be used for numerical purposes (see system (39) and associated Appendix 2).

\section{Positivity results:}

We now want to highlight some properties of positivity of the regular solutions of ((4)-(5), (10), (14)), or alternatively (15), (14). The demonstration of the following results can be found in Appendix 4 (section A).

If we assume that $U \in L^{\infty}(\Omega \times[0, T])$ and $\partial_{x}(U) \in L^{\infty}(\Omega \times[0, T])$ then,

$$
\rho \geq 0
$$

Moreover if we assume that $\rho \in L^{\infty}(\Omega \times[0, T])$ and that $P$ remains in $\left[P_{M I N}, P_{M A X}\right]$,

$$
C \leq 1
$$

and if the additional condition $\bar{C} \geq 0$ is fulfilled then:

$$
C \geq 0
$$

Nonetheless nothing ensures that $\bar{C}$ remains in $[0,1]$, nothing ensures that $P$ remains in $\left[P_{M I N}, P_{M A X}\right]$, and nothing ensures that $\rho_{l}$ will remain positive.

\section{Remark 3:}

An analytical solution of the HRM model can go out of the domain $D_{H R M}(13)$. It is thus possible for a "consistent and stable numerical approximation" to go out of $D_{H R M}$. In practice, if a numerical solution does not remain in this domain the computations will be stopped.

\section{Entropy and source terms}

In this section we wonder whether the source term $\Gamma$ may contribute to the entropy dissipation.

We consider the functions $S_{H R M}:(P, \rho, C) \mapsto S_{H R M}(P, \rho, C)$ that are in agreement with:

$$
a_{H R M}^{2} \partial_{P}\left(S_{H R M}\right)+\partial_{\rho}\left(S_{H R M}\right)=0
$$

We introduce the entropy-entropy flux pair: $\left(\eta_{H R M}, F_{\eta}^{H R M}\right)$

$$
\begin{gathered}
\eta_{H R M}=-\rho \ln \left(S_{H R M}\right) \\
F_{\eta}^{H R M}=U \eta_{H R M}
\end{gathered}
$$

Using a classical approach - that is: introducing a viscous contribution in governing equations, deriving the evolution equation of the entropy in that framework, and then passing to the limit by enforcing a vanishing viscosity (see ${ }^{19}$ for instance )- we know that smooth solutions of (4) agree with the entropy inequality:

$$
\partial_{t}\left(\eta_{H R M}\right)+\partial_{x}\left(F_{\eta}^{H R M}\right) \leq 0
$$

when no source term $\Gamma$ is present in (4). 
We now focus on the smooth solutions of the HRM model with the zero-order source term (4). These are such that (see Appendix 4, section B):

$$
\partial_{t}\left(\eta_{H R M}\right)+\partial_{x}\left(F_{\eta}^{H R M}\right)=\Gamma_{\eta}
$$

with:

$$
\Gamma_{\eta}=\Gamma \frac{\rho}{S_{H R M}} \partial_{P}\left(S_{H R M}\right)\left[\frac{\partial_{C}\left(S_{H R M}\right)}{\partial_{P}\left(S_{H R M}\right)}-\frac{\partial_{C}\left(e_{H R M}\right)}{\partial_{P}\left(e_{H R M}\right)}\right]
$$

Thus, in order to comply with the entropy inequality $(21)$, the source term $\Gamma$ should agree with the relation:

$$
\Gamma_{\eta} \leq 0
$$

In order to specify conditions on $\Gamma$, we introduce the physical entropy:

$$
S_{H R M}=\operatorname{Pf}(\rho, C)
$$

with:

$$
f(\rho, C)=f_{0}(C)\left(\left(1+\rho A_{v}\left(1-\delta_{l} / \delta_{v}\right)(1-C) /\left(\delta_{l}-1\right)\right) / \rho\right)^{\gamma_{l}}
$$

where the positive function $f_{0}(C)$ should fulfill: $f_{0}(1)=1$. Hence the above condition turns out to be :

$$
\Gamma\left[\frac{\partial_{C}\left(S_{H R M}\right)}{\partial_{P}\left(S_{H R M}\right)}-\frac{\partial_{C}\left(e_{H R M}\right)}{\partial_{P}\left(e_{H R M}\right)}\right]<0
$$

Using the expression of $S_{H R M}=\operatorname{Pf}(\rho, C)$ above, one can check that the form of the source term $\Gamma$ does not necessarily satisfy the constraint. This may be easily explained: the source term $\Gamma$ has been picked up from the standard two-phase flow literature, whatever the EOS of the mean fluid is, which may result in a potential conflict. In practice, if one retains $f_{0}(C)=1$, the agreement will occur if and only if the source term $\Gamma$ is positive. 


\section{The Homogeneous Equilibrium Model}

\section{A. Governing equations}

The Homogeneous Equilibrium Model is governed by a set of three equations in order to account for total mass, total momentum and total energy balances. These read:

$$
\left\{\begin{array}{l}
\partial_{t}(\rho)+\partial_{x}(\rho U)=0 \\
\partial_{t}(\rho U)+\partial_{x}\left(\rho U^{2}+P\right)=0 \\
\partial_{t}\left(\rho E_{H E M}\right)+\partial_{x}\left(U\left(\rho E_{H E M}+P\right)\right)=0
\end{array}\right.
$$

with:

$$
E_{H E M} \stackrel{\text { def }}{=} e_{H E M}(P, \rho)+\frac{U^{2}}{2} \text { and } e_{H E M}(P, \rho) \stackrel{\text { def }}{=} h_{H E M}(P, \rho)-\frac{P}{\rho}
$$

where, again, the function $h_{H E M}(P, \rho)$ is the specific enthalpy, and must be prescribed by the user.

We want to ensure the compatibility of this thermodynamic closure with the HRM one, in a sense to be precised later. For this reason, we can restrain the domain of validity of system (22) to:

$$
D_{H E M}=\left\{(\rho, P) / P \in\left[P_{M I N}, P_{M A X}\right], \rho>\left(\tau_{v}^{s}(P)\right)^{-1}\right\}
$$

Moreover, the thermodynamic relations for the liquid and the vapour (7), (8), (9) still hold.

First we set:

$$
C_{e q}(P, \rho) \stackrel{\text { def }}{=} \frac{\tau_{v}^{s}(P)-\tau}{\tau_{v}^{s}(P)-\tau_{l}^{s}(P)}
$$

We also need to introduce two subdomains:

- the pure liquid domain $D_{l}=\left\{\tau<\tau_{l}^{s}(P), P \in\left[P_{M I N}, P_{M A X}\right]\right\}$

- the two-phase region $D_{2 \phi}=\left\{\tau_{l}^{s}(P) \leq \tau \leq \tau_{v}^{s}(P), P \in\left[P_{M I N}, P_{M A X}\right]\right\}$.

In order to complete $(22),(23)$, we define the specific enthalpy $h_{H E M}(P, \rho)$ on the pure liquid domain and on the two-phase domain:

- Pure liquid domain $D_{l}$ : if $C_{e q}>1$ or equivalently $\tau<\tau_{l}^{s}(P)$ :

$$
h_{H E M}(P, \rho) \stackrel{\text { def }}{=} h_{l}(P, \rho)
$$

- Two-phase domain $D_{2 \phi}$ : if $0 \leq C_{e q} \leq 1$ or equivalently $\tau_{l}^{s}(P) \leq \tau \leq \tau_{v}^{s}(P)$ :

$$
h_{H E M}(P, \rho) \stackrel{\text { def }}{=} C_{e q} h_{l}\left(P, \rho_{l}\left(P, \rho, C_{e q}\right)\right)+\left(1-C_{e q}\right) h_{v}^{s}(P)
$$

Using (11) and (25), we get: $\rho_{l}\left(P, \rho, C=C_{e q}\right)=1 / \tau_{l}^{s}(P)$. Hence the enthalpy of the HEM model in the two-phase domain $D_{2 \phi}$, simply reads :

$$
h_{H E M}(P, \rho)=C_{e q} h_{l}^{s}(P)+\left(1-C_{e q}\right) h_{v}^{s}(P)
$$

The enthalpy $h_{H E M}$ is continuous at the boundary between the two domains $D_{2 \phi}$ and $D_{l}$ (i.e. continuous at $\left.C_{e q}=1\right)$. Nevertheless, its derivatives are not continuous.

In the following, the HEM model will refer to the set of equation (22), (23) with the above definitions (26) of $h_{H E M}$ and the saturation curves.

\section{Remark 4:}

It is important to note that $C_{e q}(P, \rho)=\bar{C}$. The relaxation source term (19) of the HRM model can now be written:

$$
\Gamma=(1-C)\left(C_{e q}(P, \rho)-C\right) \frac{G_{2}(P, \rho)}{\tau_{0}}
$$


Then $C=C_{e q}(P, \rho)$ is a curve for the equilibrium $\Gamma=0$.

\section{Remark 5:}

There is a compatibility of the EOS of the HEM model with the EOS of the HRM model. The relation

$$
\rho_{l}\left(P, \rho, C=C_{e q}\right)=\frac{1}{\tau_{l}^{s}(P)}
$$

implies that:

$$
h_{H E M}(P, \rho)=h_{H R M}\left(P, \rho, C=C_{e q}\right)
$$

In section IV, we study the coupling of these HEM and HRM models. The previous choice on compatible EOS will allow us to focus on the main differences between models rather than on discrepancies linked with inhomogeneities of EOS. The latter problem is addressed in. ${ }^{4,10}$

\section{B. Hyperbolicity}

In order to study the hyperbolicity of the HEM model, we focus on (22). Then the non-conservative equations on $\rho, U$ and $P$ are:

$$
\partial_{t}\left(\begin{array}{c}
\rho \\
U \\
P
\end{array}\right)+\left(\begin{array}{ccc}
U & \rho & 0 \\
0 & U & \tau \\
0 & \hat{\gamma}_{H E M} P & U
\end{array}\right) \partial_{x}\left(\begin{array}{c}
\rho \\
U \\
P
\end{array}\right)=\left(\begin{array}{l}
0 \\
0 \\
0
\end{array}\right)
$$

with:

\section{Property 3}

$$
\hat{\gamma}_{H E M}=\left(P \partial_{P}\left(e_{H E M}\right)\right)^{-1}\left(\frac{P}{\rho}-\rho \partial_{\rho}\left(e_{H E M}\right)\right)
$$

The system (28) is hyperbolic if and only if $\hat{\gamma}_{H E M} P>0$. Its eigenvalues and right eigenvectors are recalled below:

$$
\begin{array}{ll}
\lambda_{1}=U-a_{H E M} ; & r_{1}^{\top}=\left(\rho,-a_{H E M}, \rho a_{H E M}^{2}\right) \\
\lambda_{2}=U ; & r_{2}^{\top}=(1,0,0) \\
\lambda_{3}=U+a_{H E M} ; & r_{3}^{\top}=\left(\rho,+a_{H E M}, \rho a_{H E M}^{2}\right)
\end{array}
$$

where the sound velocity $a_{H E M}$ is defined by:

$$
a_{H E M} \stackrel{\text { def }}{=}\left(\hat{\gamma}_{H E M} P / \rho\right)^{1 / 2}
$$

Since the derivatives of $e_{H E M}$ are not defined in the whole domain $D_{H E M}, \hat{\gamma}_{H E M}$ is only defined inside the subdomains $D_{l}$ and $D_{2 \Phi}$.

\section{Property 4}

The HEM model is hyperbolic inside the pure liquid domain and inside the two-phase domain.

Proof

The sound speed reads:

- In the pure liquid domain: $a_{H E M}=\sqrt{\gamma_{l} P \tau}=\sqrt{\frac{\delta_{l}}{\delta_{l}-1} P} \quad \forall(\tau, P) \in \stackrel{\circ}{D_{l}}$

- In the two-phase domain the formulas are cumbersome and no sufficient condition clearly appears to ensure the positivity of $a_{H E M}^{2}$. For all couple $(\tau, P)$ in $\frac{\circ}{D_{2 \Phi}}$, we have:

$$
a_{H E M}^{2}=\frac{\left(\frac{P}{\rho}-\rho \partial_{\rho}(e)\right)}{\rho \partial_{P}(e)}
$$

with:

$$
\begin{gathered}
\left(\frac{P}{\rho}-\rho \partial_{\rho}(e)\right)=\rho \partial_{\rho}\left(C_{e q}\right)\left(h_{v}^{s}-h_{l}^{s}\right) \\
10 \text { of } 40
\end{gathered}
$$




$$
\partial_{P}(e)=-\tau+\partial_{P}\left(C_{e q}\right)\left(h_{l}^{s}-h_{v}^{s}\right)+C_{e q} \frac{d}{d P} h_{l}^{s}+\left(1-C_{e q}\right) \frac{d}{d P} h_{v}^{s}
$$

Nonetheless it can be shown that, with our choices for the saturation curves and for the chosen perfect gas coefficients, $\frac{P}{\rho}-\rho \partial_{\rho}(e)$ and $\partial_{P}(e)$ remain positive inside the two-phase domain $D_{2 \Phi}$.

\section{Some positivity results for the HEM model}

Again, if we consider smooth solutions of the HEM model (28), with positive initial conditions and -inletboundary conditions for $\rho$ and $P$, we are ensured that the mean density will remain positive, and also that the mean pressure $P$ will remain positive for $(x, t) \in \Omega \times[0, T]$ provided that $U, \hat{\gamma}_{H E M}$ and $\partial_{x}(U)$ lie in $L^{\infty}(\Omega \times[0, T])$ (for more details, see Appendix 4).

However, nothing guarantees that for smooth solutions, $\rho$ will agree with $\rho>\left(\tau_{v}^{s}(P)\right)^{-1}$ and $P$ will remain in $\left[P_{M I N}, P_{M A X}\right]$. In practice, the computation is stopped if one of these events occurs. 


\section{A method to couple HEM and HRM models}

We want to couple an "HEM" code and an "HRM" code through an interface $x=0$. The HEM domain will correspond to $x<0$, and the HRM domain to $x>0$ (see figure 1).

One of the main difficulties which arise when one aims at coupling the HRM and HEM models is due to the fact that the liquid mass fraction $C$ is governed by a PDE in the HRM, and issues from a local constraint $C=C_{e q}(P, \rho)$ in the HEM model. We propose to couple both models using a "father interface model" with an instantaneous relaxation process at the interface. $\mathrm{In}^{26}$, a similar approach was used to couple a one dimensional code and a two dimensional code, following ideas from ${ }^{21}$. In the present approach, we rather choose to proceed as $\mathrm{in}^{2,4}$. The main advantage is that one can get rid of the possible resonance phenomenon, since the set of right eigenvectors of the model we propose spans the whole phase space.

\section{A. The interface model}

We first rewrite the HEM set of equations. This is achieved introducing a PDE with a source term containing an instantaneous relaxation term:

$$
\partial_{t}(\rho C)+\partial_{x}(\rho U C)=\mu \rho\left(C_{e q}(P, \rho)-C\right)
$$

The set of equations for the HEM model (using the confusing notation E instead of $E_{H E M}$ on purpose) thus writes (for $x \in \mathbf{R}^{-}$and $t \in \mathbf{R}^{+}$):

$$
\left\{\begin{array}{l}
\partial_{t}(\rho C)+\partial_{x}(\rho U C)=\mu \rho\left(C_{e q}(P, \rho)-C\right) \\
\partial_{t}(\rho)+\partial_{x}(\rho U)=0 \\
\partial_{t}(\rho U)+\partial_{x}\left(\rho U^{2}+P\right)=0 \\
\partial_{t}(\rho E)+\partial_{x}(U(\rho E+P))=0
\end{array}\right.
$$

with the parameter $\mu>0$. We also recall that the governing equations for $(x, t) \in \mathbf{R}^{+} \times \mathbf{R}^{+}$are given by the HRM model (4), (5), (10).

We may now introduce the "father model". For that purpose we define a new variable $Y(x, t)$ depending on time and space, which is usually called the "color variable". The "father model" is governed by the following set of equations (for $x \in \mathbf{R}$ and $t \in \mathbf{R}^{+}$):

$$
\left\{\begin{array}{l}
\partial_{t}(\rho C)+\partial_{x}(\rho C U)=\rho T \\
\partial_{t}(\rho)+\partial_{x}(\rho U)=0 \\
\partial_{t}(\rho U)+\partial_{x}\left(\rho U^{2}+P\right)=0 \\
\partial_{t}(\rho E)+\partial_{x}(U(\rho E+P))=0 \\
\partial_{t}(\rho Y)+\partial_{x}(\rho U Y)=\lambda\left(Y_{0}-Y\right)
\end{array}\right.
$$

with:

and

$$
E=Y e_{H R M}(P, \rho, C)+(1-Y) e_{H E M}(P, \rho)+\frac{u^{2}}{2}
$$

$$
T=Y \Gamma+(1-Y) \mu\left(C_{e q}-C\right)
$$

At the time $t=0$ we initialize $Y(x, 0)=Y_{0}(x)$ such that $Y_{0}(x)=1$ if $x$ is in the HRM domain and $Y_{0}(x)=0$ if $x$ is in the HEM domain. In the following the parameters $\lambda$ and $\mu$ will formally be set to $+\infty$ (instantaneous relaxation).

We may define a sound speed $a$ for this interface model, which reads:

$$
a^{2}=\frac{a_{H R M}^{2} Y \partial_{P}\left(e_{H R M}\right)+a_{H E M}^{2}(1-Y) \partial_{P}\left(e_{H E M}\right)}{Y \partial_{P}\left(e_{H R M}\right)+(1-Y) \partial_{P}\left(e_{H E M}\right)}
$$

\section{Property 5}

The convective part of system (32) is hyperbolic if $(C, \rho, P) \in D_{H R M}$ and $(\rho, P) \in D_{H E M}$. 
Proof

Starting from (32), and focusing on regular solutions, the convective part of the first three equations and the fifth equation may be rewritten as follows:

$$
\left\{\begin{array}{l}
\partial_{t}(C)+U \partial_{x}(C)=0 \\
\partial_{t}(\rho)+U \partial_{x}(\rho)+\rho \partial_{x}(U)=0 \\
\partial_{t}(U)+U \partial_{x}(U)+\tau \partial_{x}(P)=0 \\
\partial_{t}(Y)+U \partial_{x}(Y)=0
\end{array}\right.
$$

Meanwhile, the total energy equation is equivalent to:

$$
\left\{\begin{array}{l}
\left(Y \partial_{P}\left(e_{H R M}\right)+(1-Y) \partial_{P}\left(e_{H E M}\right)\right)\left(\partial_{t}(P)+U \partial_{x}(P)\right) \\
+\left(Y \partial_{\rho}\left(e_{H R M}\right)+(1-Y) \partial_{\rho}\left(e_{H E M}\right)\right)\left(\partial_{t}(\rho)+U \partial_{x}(\rho)\right)+(P / \rho) \partial_{x}(U)=0
\end{array}\right.
$$

Thus, using definitions of $a_{H R M}$ and $a_{H E M}$ from (17) and (29), the definition of $a$ given by (33) and applying (34) enables to derive the governing equation for the mean pressure as:

$$
\partial_{t}(P)+U \partial_{x}(P)+\rho a^{2} \partial_{x}(U)=0
$$

We know from the study of the HEM and HRM models that $\partial_{P}\left(e_{H R M}\right), a_{H R M}^{2}$ are positive if $(C, \rho, P)$ is in the domain $D_{H R M}$, and that $\partial_{P}\left(e_{H E M}\right)$, and $a_{H E M}^{2}$ are positive if $(\rho, P)$ lie in $D_{H E M}$. Furthermore, the system (32) ensures that if $Y_{0}$ is in $[0,1], Y$ remains in $[0,1]$. Hence $a^{2}$ is positive. Therefore, a straightforward and classic calculation enables to exhibit eigenvalues:

$$
\lambda_{1,2,3}=U, \quad \lambda_{4}=U-a, \quad \lambda_{5}=U+a
$$

One may easily check that the associated right eigenvectors span $\mathbf{R}^{5}$. We may then conclude that the convective part of system (32) is hyperbolic if $(C, \rho, P) \in D_{H R M}$ and $(\rho, P) \in D_{H E M}$.

\section{B. Numerical scheme}

All methods described herein rely on the Finite Volume method $\left({ }^{12,19}\right)$. We restrict here to the so-called "first-order" schemes. As an approximate Godunov scheme, we will apply VFRoe-ncv using the non conservative variable $(\rho, U, P)$ for the HEM system and $(\rho, U, P, C)$ for the HRM system (see ${ }^{7,15}$ for details on these approximate Godunov schemes). The coupling interface model which has been introduced above will be used to compute "fluxes" at the interface between the two domains.

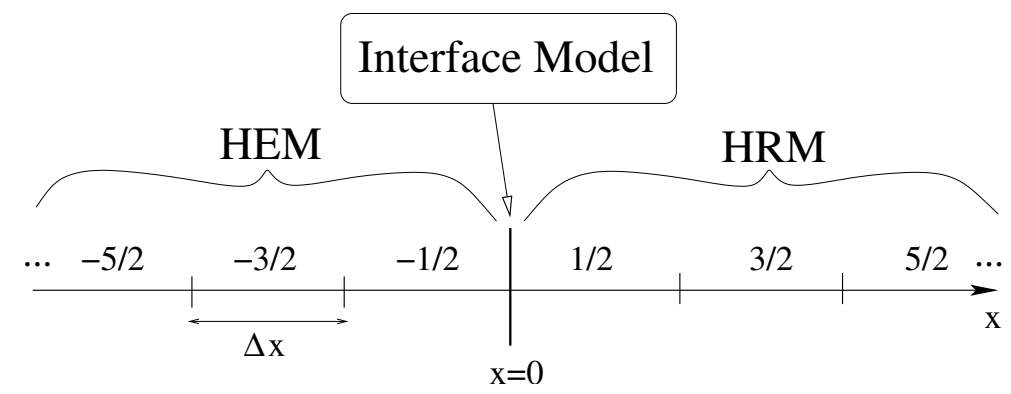

Figure 1. Notations and models.

Let us introduce a fixed uniform mesh space $\Delta x$ and a time step $\Delta t$ in agreement with a CFL condition. We set for all integers $j$ and $n$,

$$
x_{j+1 / 2}=\left(j+\frac{1}{2}\right) \Delta x, \quad x_{j}=j \Delta x, \quad t^{n}=n \Delta t
$$

The cell $j+1 / 2$ is delimited by the interface $j$ located in $x_{j}$ and the interface $j+1$ located in $x_{j+1}$, and its center is denoted by $x_{j+1 / 2}$. The interface $j=0$ is the coupling interface. The variable $W_{j+1 / 2}^{n}$ stands for 
the variable $W$ at the time $t^{n}$ in the cell $j+1 / 2$. The HEM domain (and the HRM domain respectively) is composed of all the cells $j+1 / 2<0$ (respectively of all the cells $j+1 / 2>0$, see figure 1 ). The conservative variables will be denoted by $W_{H E M}=\left(\rho, \rho U, \rho E_{H E M}\right)^{\top}$ in the HEM domain, $W_{H R M}=\left(\rho C, \rho, \rho U, \rho E_{H R M}\right)^{\top}$ in the HRM domain and by $W_{I N T}=(\rho C, \rho, \rho U, \rho E, \rho Y)^{\top}$ for the interface model.

We assume that $Y_{j+1 / 2}^{n}=0$ for all $j+1 / 2<0$ and $Y_{j+1 / 2}^{n}=1$ for all $j+1 / 2>0$ at a discrete time $t^{n}$. The numerical scheme which is used to compute the variable at time step $t^{n+1}=t^{n}+\Delta t$ contains three steps:
1) A convection step, $t^{n} \rightarrow t^{\tilde{n}}$.
2) An instantaneous relaxation on $Y, t^{\tilde{n}} \rightarrow t^{\hat{n}}$.
3) A finite relaxation step, $t^{\hat{n}} \rightarrow t^{n+1}$.

1. The convection step, $t^{n} \rightarrow t^{\tilde{n}}$

This step of the scheme does not take into account the different source terms. The fluxes are computed at each interface $j$.

(i) For all interface $j \leq-1$ (HEM domain) we apply the VFRoe-ncv scheme using the variable $(\rho, U, P)^{\top}$ on the convective part of the system (22). The flux is denoted $F_{H E M, j}^{n}$.

(ii) For all interface $j \geq 1$ (HRM domain) we apply VFRoe-ncv scheme using the variable $(C, \rho, U, P)^{\top}$ on the convective part of the system (4). The flux is denoted $F_{H R M, j}^{n}$.

(iii) At $j=0$ (the coupling interface) the flux is built with a modified VFRoe-ncv scheme which uses the variable $(C, \rho, U, P, Y)^{\top}$ in order to compute approximate interface states in the solution of the onedimensional Riemann problem associated with the system (32). The left state in the initial condition of the Riemann problem is

$$
\left(\left(\rho C_{e q}(P, \rho)\right)_{-1 / 2}^{n}, \rho_{-1 / 2}^{n},(\rho U)_{-1 / 2}^{n},\left(\rho E_{H E M}\right)_{-1 / 2}^{n},(\rho Y)_{-1 / 2}^{n}\right)^{\top}
$$

and the right state in the initial condition is

$$
\left((\rho C)_{1 / 2}^{n}, \rho_{1 / 2}^{n},(\rho U)_{1 / 2}^{n},\left(\rho E_{H R M}\right)_{1 / 2}^{n},(\rho Y)_{1 / 2}^{n}\right)^{\top}
$$

The flux is denoted $F_{I N T}^{n}$. Details on the computation of $F_{I N T}^{n}$ are given in Appendix 1 .

Once the fluxes have been computed for all interfaces, the value of the cell is updated using the formula:

- If $j+1 / 2 \leq-3 / 2$

$$
W_{H E M, j+1 / 2}^{\tilde{n}}-W_{H E M, j+1 / 2}^{n}+\frac{\Delta t}{\Delta x}\left(F_{H E M, j}^{n}-F_{H E M, j-1}^{n}\right)=0
$$

- If $j+1 / 2 \geq 3 / 2$

$$
W_{H R M, j+1 / 2}^{\tilde{n}}-W_{H R M, j+1 / 2}^{n}+\frac{\Delta t}{\Delta x}\left(F_{H R M, j}^{n}-F_{H R M, j-1}^{n}\right)=0
$$

- If $j+1 / 2=-1 / 2$

$$
\left(\begin{array}{l}
W_{H E M} \\
\rho Y
\end{array}\right)_{-1 / 2}^{\tilde{n}}-\left(\begin{array}{l}
W_{H E M} \\
\rho Y
\end{array}\right)_{-1 / 2}^{n}+\frac{\Delta t}{\Delta x}\left(\left(F_{2-5}\right)_{I N T}^{n}-\left(\begin{array}{l}
F_{H E M,-1}^{n} \\
(\rho U Y)_{H E M,-1}^{n}
\end{array}\right)\right)=0
$$

The flux $\left(F_{2-5}\right)_{I N T}^{n}$ is composed of the second, third, fourth and fifth components of $F_{I N T}^{n}$

- If $j+1 / 2=1 / 2$

$$
\left(\begin{array}{l}
W_{H R M} \\
\rho Y
\end{array}\right)_{1 / 2}^{\tilde{n}}-\left(\begin{array}{l}
W_{H R M} \\
\rho Y
\end{array}\right)_{1 / 2}^{n}+\frac{\Delta t}{\Delta x}\left(\left(\begin{array}{l}
F_{H R M, 1}^{n} \\
(\rho U Y)_{H R M, 1}^{n}
\end{array}\right)-F_{I N T}^{n}\right)=0
$$

An important point to underline is that: $Y_{j+1 / 2}^{\tilde{n}}=Y_{j+1 / 2}^{n}$ in all the cells, except in the cell $j=1 / 2$ if $U_{I N T}^{n}>0$ and in the cell $j=-1 / 2$ if $U_{I N T}^{n}<0$. 
2. Instantaneous relaxation step, $t^{\tilde{n}} \rightarrow t^{\hat{n}}$

For this step we consider the ODE system on the whole domain $(x \in \mathbb{R})$ :

$$
\left\{\begin{array} { l } 
{ \partial _ { t } ( \rho C ) = 0 } \\
{ \partial _ { t } ( \rho ) = 0 } \\
{ \partial _ { t } ( \rho U ) = 0 } \\
{ \partial _ { t } ( \rho E ) = 0 } \\
{ \partial _ { t } ( \rho Y ) = \lambda ( Y _ { 0 } - Y ) \text { with } \lambda = + \infty }
\end{array} \Longleftrightarrow \left\{\begin{array}{l}
\partial_{t}(\rho C)=0 \\
\partial_{t}(\rho)=0 \\
\partial_{t}(\rho U)=0 \\
\partial_{t}(\rho E)=0 \\
Y=Y_{0}
\end{array}\right.\right.
$$

This can be rewritten in terms of discrete variables (for all $k$ ) as:

$$
\left\{\begin{array} { l } 
{ ( \rho C ) _ { k } ^ { \hat { n } } = ( \rho C ) _ { k } ^ { \tilde { n } } } \\
{ \rho _ { k } ^ { \hat { n } } = \rho _ { k } ^ { \tilde { n } } } \\
{ ( \rho U ) _ { k } ^ { \hat { n } } = ( \rho U ) _ { k } ^ { \tilde { n } } } \\
{ ( \rho E ) _ { k } ^ { \hat { n } } = ( \rho E ) _ { k } ^ { \tilde { n } } } \\
{ Y _ { k } ^ { \hat { n } } = Y _ { k } ^ { 0 } }
\end{array} \quad \text { and thus } \quad \left\{\begin{array}{l}
C_{k}^{\hat{n}}=C_{k}^{\tilde{n}} \\
\rho_{k}^{\hat{n}}=\rho_{k}^{\tilde{n}} \\
U_{k}^{\hat{n}}=U_{k}^{\tilde{n}} \\
e_{k}^{\hat{n}}=e_{k}^{\tilde{n}} \\
Y_{k}^{\hat{n}}=Y_{k}^{0}
\end{array}\right.\right.
$$

This step is totally transparent with respect to physical conservative variables. Let us call $k$ the cell $j+1 / 2$ for which $Y_{j+1 / 2}^{\tilde{n}} \neq Y_{j+1 / 2}^{n}$, for all the other cells we have $Y_{j+1 / 2}^{\tilde{n}}=Y_{j+1 / 2}^{0}$. The fourth equation of (37) implies that the pressure changes in the cell $k$ during the current step. Indeed equations (37) imply:

$$
e\left(P_{k}^{\hat{n}}, \rho_{k}^{\tilde{n}}, C_{k}^{\tilde{n}}, Y_{k}^{\hat{n}}\right)=e\left(P_{k}^{\tilde{n}}, \rho_{k}^{\tilde{n}}, C_{k}^{\tilde{n}}, Y_{k}^{\tilde{n}}\right)
$$

Thus $Y_{k}^{\hat{n}} \neq Y_{k}^{\tilde{n}}$ implies that $P_{k}^{\hat{n}} \neq P_{k}^{\tilde{n}}$.

3. The finite time relaxation step, $t^{\hat{n}} \rightarrow t^{n+1}$

In this final step we compute the source term $T$. In the HEM domain (that is: $x<0$ ), this simply corresponds to the following update:

$$
C^{n+1}=C_{e q}\left(P^{\hat{n}}, \rho^{\hat{n}}\right)
$$

In the HRM domain (that is: $x>0$ ), it urges to find approximations of

$$
\left\{\begin{array}{l}
\partial_{t}(\rho C)=\rho \Gamma \\
\partial_{t}(\rho)=0 \\
\partial_{t}(\rho U)=0 \\
\partial_{t}(\rho E)=0
\end{array}\right.
$$

In terms of the non-conservative variables $(C, \rho, U, P)$, and restricting to regular solutions, (39) is equivalent to:

$$
\left\{\begin{array}{l}
\partial_{t}(C)=\Gamma \\
\partial_{t}(\rho)=0 \\
\partial_{t}(U)=0 \\
\partial_{t}(P)=-\left(\partial_{P}\left(e_{H R M}\right)\right)^{-1} \partial_{C}\left(e_{H R M}\right) \Gamma
\end{array}\right.
$$

The time scheme which is used to discretize (40) is detailed in Appendix 2. It provides $C^{n+1}$ and $P^{n+1}$, while we get $\rho^{n+1}=\rho^{\hat{n}}$ and $U^{n+1}=U^{\hat{n}}$. The final update of conservative variables is achieved through:

$$
\left\{\begin{array}{l}
(\rho C)^{n+1}=\rho^{\hat{n}} C^{n+1} \\
\rho^{n+1}=\rho^{\hat{n}} \\
(\rho U)^{n+1}=(\rho U)^{\hat{n}} \\
(\rho E)^{n+1}=\rho^{\hat{n}}\left(e_{H R M}\left(P^{n+1}, \rho^{\hat{n}}, C^{n+1}, Y^{\hat{n}}\right)+\frac{\left(U^{\hat{n}}\right)^{2}}{2}\right) \\
(\rho Y)^{n+1}=(\rho Y)^{\hat{n}}
\end{array}\right.
$$




\section{Remark 6:}

- The convection step (thanks to the VFRoe-ncv scheme and the respect of the CFL condition) and the instantaneous relaxation step maintain the discrete liquid mass fraction $C^{\hat{n}}$ in $[0,1]$. Moreover, if the discrete values of the equilibrium liquid mass fraction $C_{e q}$ remain in $[0,1]$ during the finite time relaxation computation, $C^{n+1}$ will remain in [0,1] (see Appendix 2).

- The introduction of the fictitious variable $Y$ does not lead to any modification of the left and right codes. Actually, the convection step implicitly provides boundary conditions on both sides of the coupling interface. 


\section{Numerical results}

Many test cases involving single waves, and in particular contact waves enable us to check that numerical methods used within each code are well-suited. More precisely, the exact analytical form of the internal energy, for both the HEM and the HRM models, is such that the approximate Godunov scheme VFRoe-ncv (using variables $U, P, \rho$ and $U, P, \rho, C$ for HEM and HRM models respectively) perfectly preserves these pure contact waves on coarse and fine meshes $\left(\operatorname{see}^{14}\right)$. This is still true for the coupled HEM/HRM model, for similar reasons (see Appendix 3).

The different parameters will be set as follows:

$$
\begin{gathered}
P_{M I N}=0.110^{5} \mathrm{~Pa} \text { and } P_{M A X}=20010^{5} \mathrm{~Pa} \\
\gamma_{l}=1.001 \quad \text { and } \quad \gamma_{v}=1.4
\end{gathered}
$$

The form of the saturation curves is obtained using the following numerical values:

$$
h_{l}^{s}=A_{l} P+B_{l} \quad h_{v}^{s}=A_{v} P+B_{v}
$$

with the coefficients:

$$
A_{l}=8.1810^{-2} \quad B_{l}=1.9110^{5} \quad A_{v}=-8.3210^{-3} \quad B_{v}=2.5810^{6}
$$

The whole data correspond to nuclear cooling conditions.

We successively consider two different test cases which correspond to:

- the computation of Riemann problems including shock waves, contact waves and rarefaction waves,

- a schematic computation of the flow in the coolant circuit of a nuclear power plant.

\section{A. Riemann problems}

We consider in this section a computational domain : $x \in[-1,1]$ which is discretized using a uniform mesh. We will compare for different mesh sizes, the results obtained with:

- The Homogeneous Equilibrium Model over the whole computational domain,

- The Homogeneous Relaxation Model over the whole computational domain,

- The coupling configuration in which HEM is used for $x<0, \mathrm{HRM}$ is used for $x>0$, and the coupling technique described in section IV is applied for $x=0$.

Hence, we will be able to compare the results of the coupled case with those obtained with a "reference solution", which will correspond with either the HRM results (or the HEM results) computed on a very fine mesh. In the nuclear community, the most widely used reference is the HRM model.

We focus here on test cases that are based on Riemann problems, where the initial discontinuity will be located either in the HEM code $(x=-0.25)$, or in the HRM code $(x=+0.25)$, as described in figures 2 and 3. The main goal here is to observe the behavior of transmitted and reflected waves through the interface $x=0$, and the numerical pollution due to the coupling scheme.

We emphasize that :

- Three different meshes will be used. An "industrial" mesh with 100 cells (fifty cells within each code), a medium size mesh with 500 cells, and a fine mesh including 10000 cells that will provide "almost converged" solutions.

- Rarefaction waves will be investigated, with special emphasis on the reflected and transmitted waves through the coupling interface. The counterpart including the propagation of shock waves through the coupling interface is reported $\mathrm{in}^{27}$. Rarefaction waves are of special interest in the nuclear framework, since the smooth solution corresponds to the Loss Of Coolant Accident (LOCA).

\section{7 of 40}




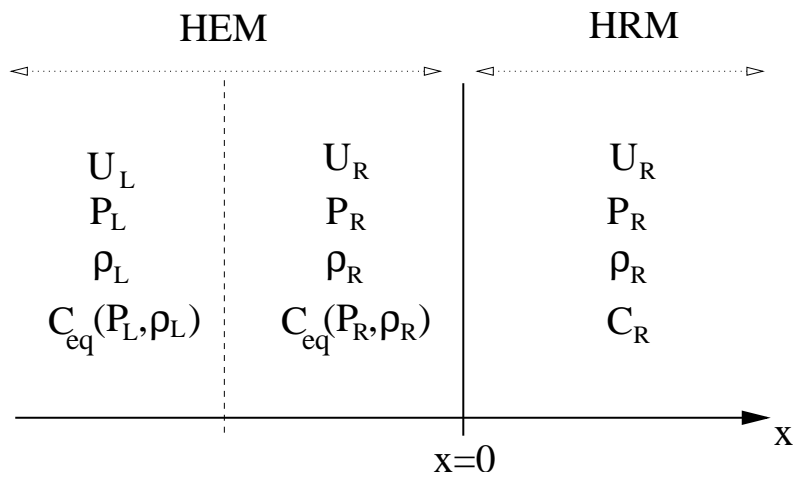

Figure 2. First configuration for initial data, the initial discontinuity is in the HEM domain

HEM HRM

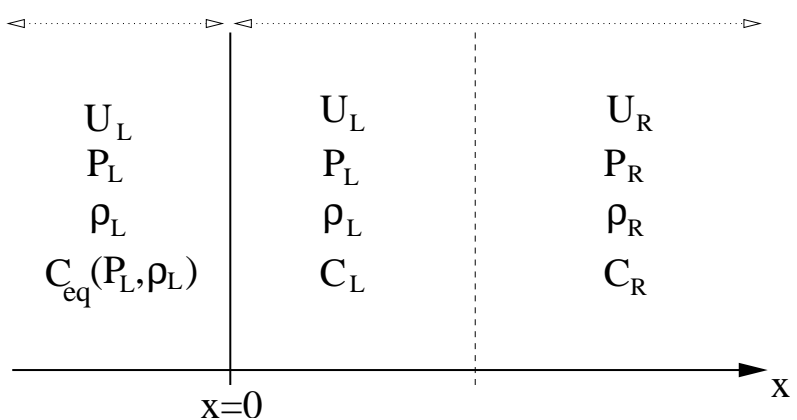

Figure 3. Second configuration for initial data, the initial discontinuity is in the HRM domain

- The time relaxation parameter $\tau_{0}=10^{-3}$ will be used. (similar tests with a different time scale $\tau_{0}=1$ are reported $\mathrm{in}^{27}$ ). In fact, when $\tau_{0}$ decreases, the similarity in the behaviour of the HRM and the HEM models improves.

In each case, two series of results are shown:

- We have gathered computations for the HEM model, HRM model and the HEM/HRM simulation on coarse meshes in order to highlight the influence of the coupling in industrial configuration (see figures 4 and 6$)$.

- Fine mesh computations for the HEM model and HRM model have been performed. They are plotted together with the results of the HEM/HRM simulation for all the meshes on the figure 5 and 7.

Hence, results include (see figures 4, 6):

- a computation of the HEM model on the whole domain, using a mesh with 100 cells (circles);

- a computation of the HRM model on the whole domain, using a mesh with 100 cells (triangles);

- a computation of the coupled case HEM $(x<0)$-HRM $(x>0)$, using a mesh with 100 cells (plain line).

A comparison is also made between results for (see figures 5,7 ):

- the "converged" HEM model on the whole domain, using a mesh with 10000 cells (circles);

- the "converged" HRM model on the whole domain, using a mesh with 10000 cells (triangles);

18 of 40

A method to couple HEM and HRM two-phase flow models 
- the "converged" coupled case HEM $(x<0)$-HRM $(x>0)$, using a mesh with 10000 cells (plain line);

- the coupled case HEM $(x<0)$-HRM $(x>0)$, using coarse meshes with 100, 500 cells ( dotted line, dashed line).

In each case we plot numerical results before the contact discontinuity hits the coupling interface $x=0$. The time step agrees with the classical CFL condition. The implicit treatment of source terms implies no additional constraint. Numerical experiments include:

- a rarefaction wave travelling from the HEM code to the HRM code;

- a rarefaction wave travelling from the HRM code to the HEM code

The dual case of shock waves is investigated $i^{27}$, and the last industrial test case contains such a configuration.

19 of 40 
1. Test 1 : A rarefaction wave traveling from HEM domain to HRM domain

Initial conditions are :

\begin{tabular}{|l|c|c|c|}
\hline domain $:$ & HEM & HEM & HRM \\
\hline \hline$U$ & 0 & 0 & 0 \\
\hline$P$ & $158 \mathrm{e} 5$ & $160 \mathrm{e} 5$ & $160 \mathrm{e} 5$ \\
\hline$\rho$ & 990 & 1000 & 1000 \\
\hline$C$ & none & none & 0.979212418 \\
\hline \hline$C_{e q}$ & 0.979261526 & 0.979212418 & 0.979212418 \\
\hline
\end{tabular}

The initial discontinuity has been set at $x=-0.25$, that is inside the HEM domain. A rarefaction wave travels towards the right and goes through the coupling interface $(x=0)$, a shock wave travels to the left, and is followed by a left-going contact discontinuity. Results have been plotted at time $t=0.025$. We notice that for the HEM computation, the whole right-going rarefaction wave has passed the coupling interface at that time.

Associated results for the sole HRM model are indeed rather close to the solutions computed with the sole HEM model. Turning then to the coupled situation, we note that the rarefaction wave coming from the HEM code does not introduce too much pollution when passing through the coupling interface, though one can notice a sharp behaviour of the pressure field around $x=0$, when the mesh is too coarse (100 cells, corresponding to the plain line on figure 4). In the coupled computation, the approximate solution in the HRM domain (respectively in the HEM domain) tends to stick immediately to the pure HRM solution (respectively to the pure HEM solution).

Another point seems worth being underlined. On the coarser mesh, one can notice (see figure 4 and 5 for instance) a sharp variation on velocity and density profiles which is located around the steady interface $x=0$. This pollution tends to decrease and clearly moves away from this interface when the mesh is refined.
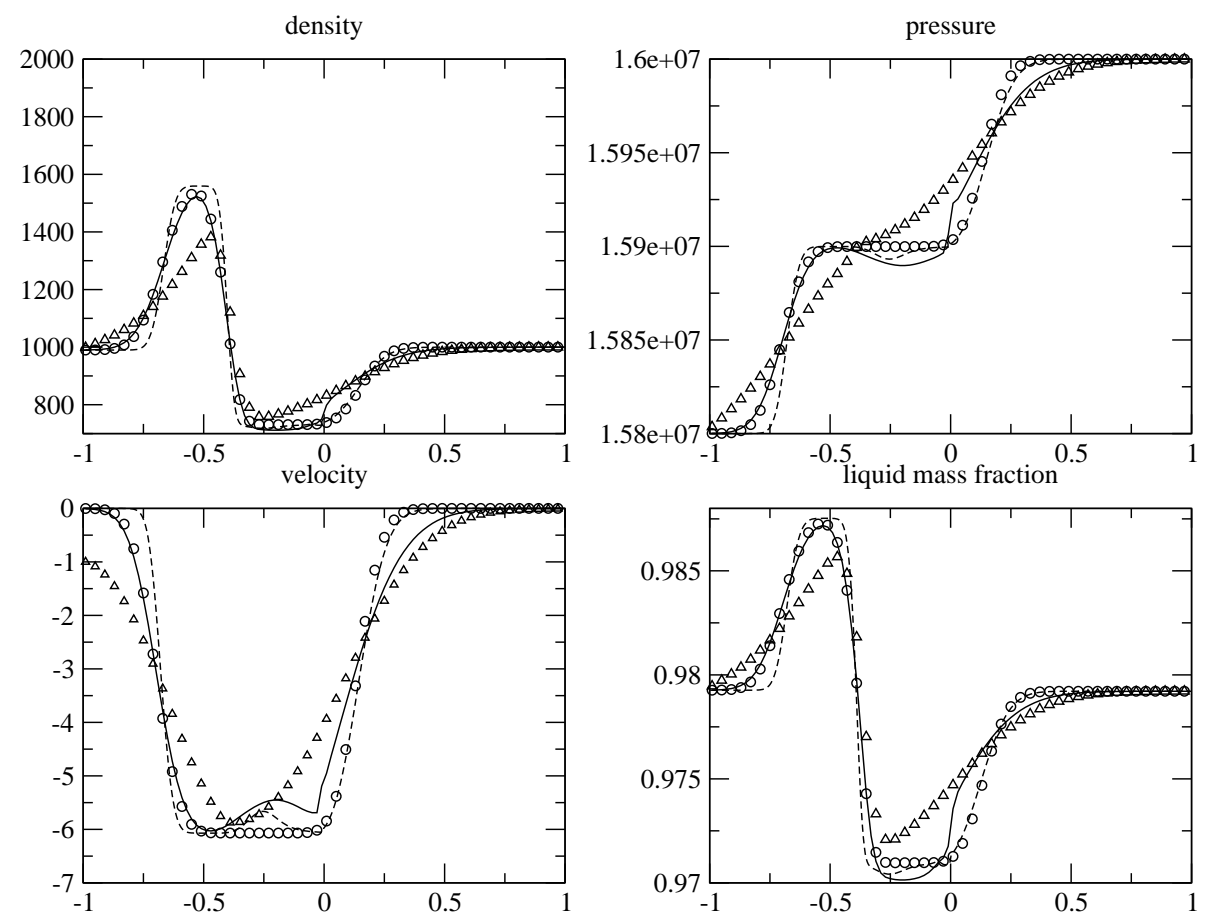

Figure 4. Test 1. Constant time scale $\tau_{0}=10^{-3}$. Circles $=$ HEM 100 cells, triangles $=$ HRM 100 cells, line $=$ HEM $/$ HRM 100 cells, dashed line $=$ HEM $/$ HRM 500 cells. 

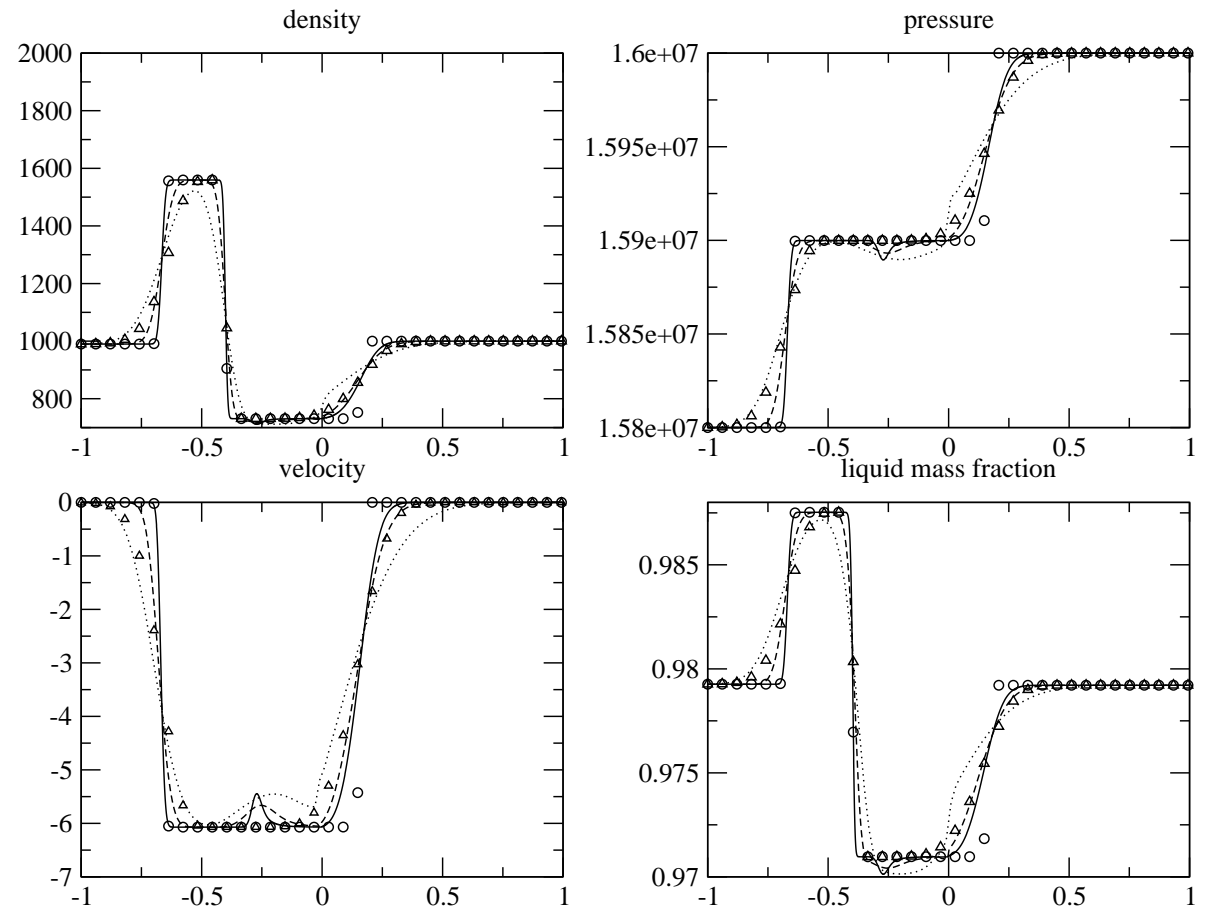

Figure 5. Test 1. Constant time scale $\tau_{0}=10^{-3}$. Circles $=$ HEM 10000 cells, triangles $=$ HRM 10000 cells, line $=$ HEM $/$ HRM 10000 cells, dashed line $=$ HEM/HRM 500 cells, dot line $=$ HEM $/$ HRM 100 cells. 
2. Test 2: A rarefaction wave traveling from HRM domain to HEM domain

Initial conditions are now the following:

\begin{tabular}{|l|c|c|c|}
\hline domain $:$ & HEM & HRM & HRM \\
\hline \hline$U$ & 0 & 0 & 0 \\
\hline$P$ & $160 \mathrm{e} 5$ & $160 \mathrm{e} 5$ & $158 \mathrm{e} 5$ \\
\hline$\rho$ & 1000 & 1000 & 990 \\
\hline$C$ & none & 0.979212418 & 0.979261526 \\
\hline \hline$C_{e q}$ & 0.979212418 & 0.979212418 & 0.979261526 \\
\hline
\end{tabular}

The initial discontinuity is now in the HRM domain, and is located at $x=0.25$. A rarefaction wave travels to the left and goes through the coupling interface $(x=0)$. Meanwhile a shock wave travels to the right boundary, and is followed by a contact discontinuity that travels towards the right. Results are still plotted at time $t=0.025$. At that time, the left-going rarefaction wave is entirely in the HEM domain in the sole HEM computation.

Remarks pertaining to the previous test case still hold. The mesh refinement helps much to improve the accuracy of the approximate solution in each subdomain. In the coupled simulation, the approximate solution is much more monotone around the coupling interface when the mesh is refined (see figure 7).
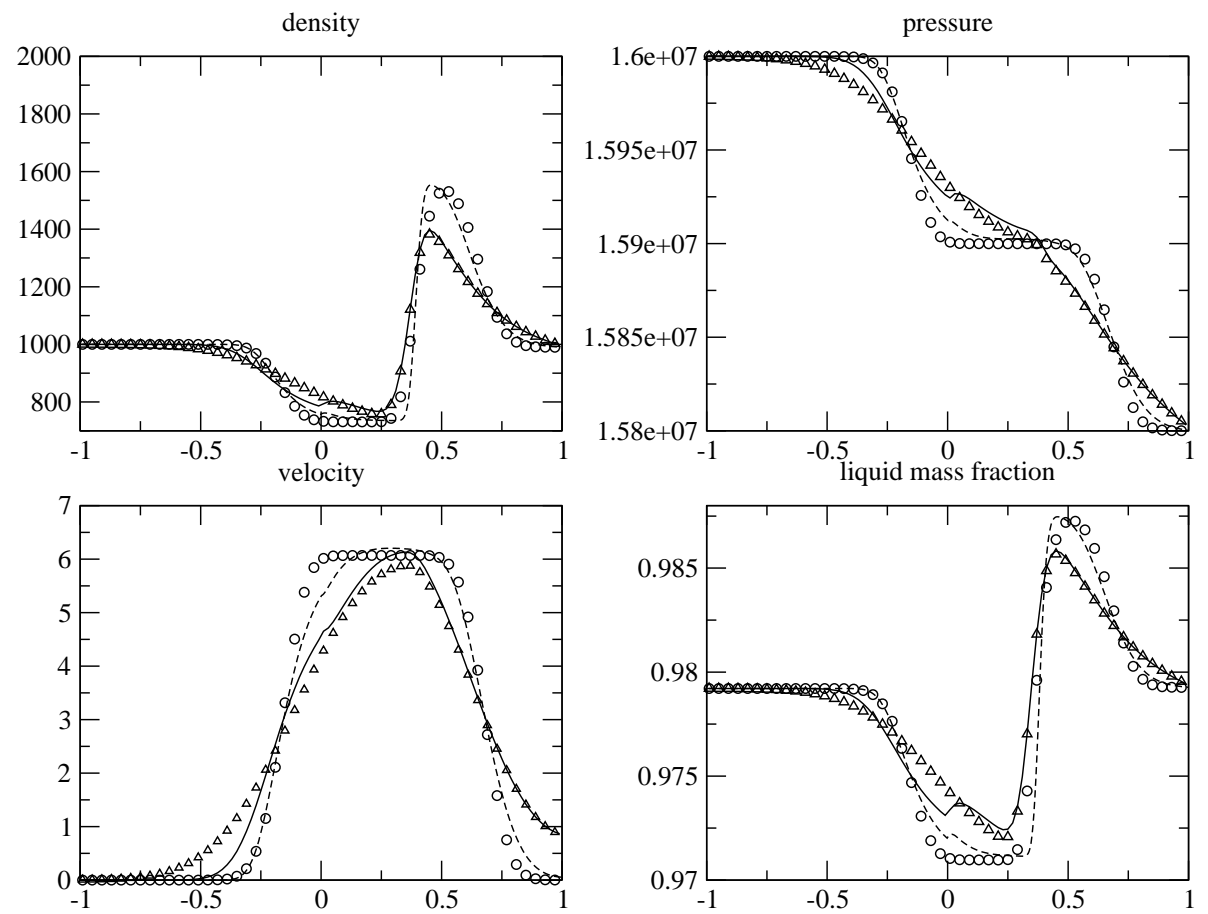

Figure 6. Test 2. Constant time scale $\tau_{0}=10^{-3}$. Circles=HEM 100 cells, triangles=HRM 100 cells, line $=$ HEM $/$ HRM 100 cells, dashed line $=$ HEM $/$ HRM 500 cells. 

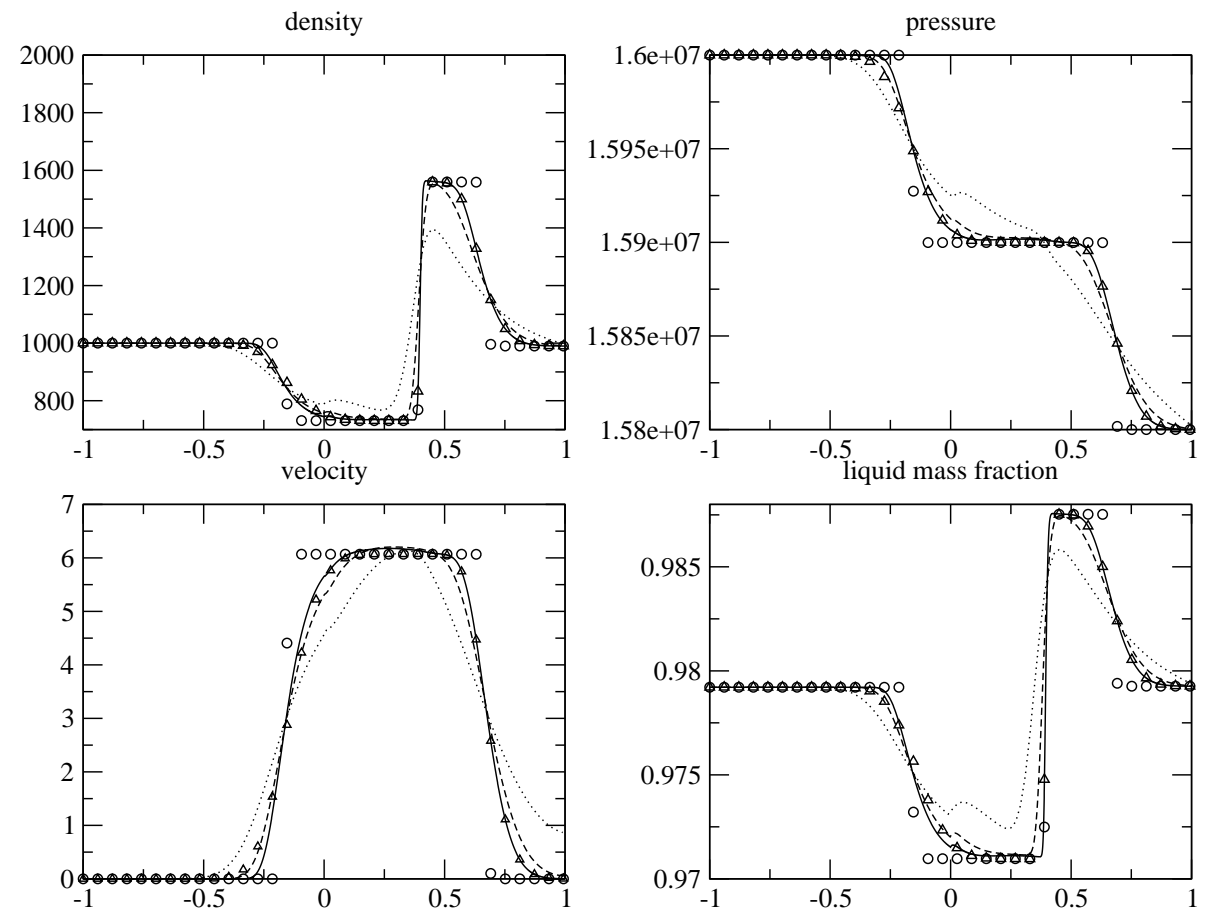

Figure 7. Test 2. Constant time scale $\tau_{0}=10^{-3}$. Circles $=$ HEM 10000 cells, triangles $=$ HRM 10000 cells, line $=$ HEM $/$ HRM 10000 cells, dashed line $=$ HEM/HRM 500 cells, dot line $=$ HEM $/$ HRM 100 cells. 


\section{B. Test 3 : A test case in a nuclear power plant}

The next test case corresponds to the schematic one dimensional flow in a nuclear power plant. The length of the pipes is $80 \mathrm{~m}$. It includes a part of the coolant circuit containing the core on the left side of the coupling interface (i.e. $-40<x<0$ ) where the governing set of equations is the HEM model. The core is located in the interval $[-22,-18]$. The total length of the core is the true core length (buoyancy effects can be neglected). The core section is equal to $3.5 \mathrm{~m}^{2}$. The HRM model is used on the right side of the coupling interface (i.e. $0<x<40$ ). Before the beginning of the computation, the velocity, density and pressure profiles are uniform and equal to: $U=10 \mathrm{~ms}^{-1}, \rho=700 \mathrm{kgm}^{-3}, P=15010^{5} \mathrm{~Pa}$; moreover $C=C_{e q}(P, \rho)$ in the HRM part of the coolant circuit. The fluid is not heated before the beginning of the computation, and the core is suddenly plugged in the coolant circuit at that time. Then the heat source $\Phi(x, t>0)$ is equal to 0 outside the core. Within the core region $(x \in[-22,-18])$, and for $t>0$, the uniform local heat source is $\Phi(x, t)=\Phi_{0}=300.10^{6} / 4$. Thus, within the core region, the total heat power is $300 \times 10^{6} \mathrm{~W}$.

In this particular test case the constants $A_{v}, B_{v}, A_{l}$, and $B_{l}$ have been estimated with $P_{M I N}=14010^{5} \mathrm{~Pa}$ and $P_{M A X}=16010^{5} \mathrm{~Pa}$ using the Thetis software ${ }^{30}$. The uniform mesh contains 500 nodes. The governing set of equations in the left part is the set of equations of the HEM model (22) with an equation for the total energy which takes the heat source into account:

$$
\partial_{t}(\rho E)+\partial_{x}(U(\rho E+P))=\Phi(x, t)
$$

Results have been plotted at four distinct instants.

- At $t=T_{1}$, the right-going shock wave, which is coming from the right side of the core, and which is due to the sudden heating in the core region, has not hit the coupling interface yet (dashed line on figure 8).

- At $t=T_{2}$, the same right-going shock wave has already moved through the coupling interface (plain line on figure 8 ). One can easily observe at $t=T_{2}$ the reflected wave in the interval $[-10,0]$, either focusing on the pressure profile, the density profile or the velocity profile. The amplitude of this reflected wave is small compared with the one of the associated shock wave.

- At $t=T_{3}$, the right-going contact wave has not passed the coupling interface yet (dashed line on figure 9). This contact wave is of course characterized by locally uniform pressure and velocity profiles. At that time, the right going shock wave has not reached the right exit. The behaviour of the coupling interface seems almost perfect, which is in agreement with test cases involving a contact wave travelling from the HEM domain towards the HRM domain $\left(\mathrm{see}^{27}\right)$.

- At $t=T_{4}$, the contact wave is on the right side of the coupling interface $(x=0)$ (plain line on figure 9 ). Once again, everything is correct around the coupling interface $(x=0)$. At that time $t=T_{4}$, the right-going shock wave has gone outside the computational domain. One can notice the influence of the right exit boundary which generates a reflected wave in the computational domain. This is due to the fact that crude "Neumann-type" boundary conditions have been imposed in this test case. 

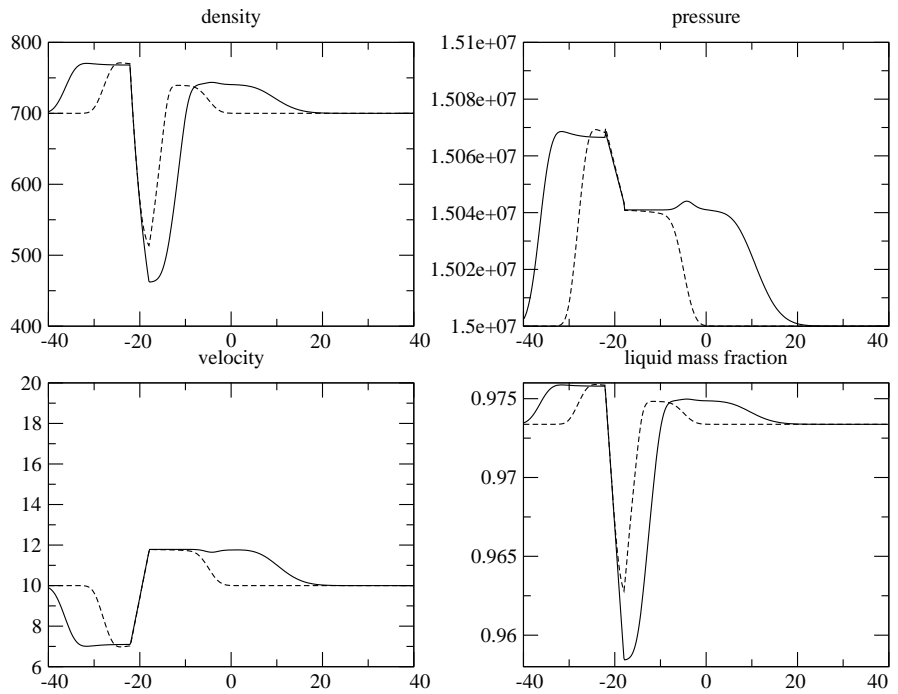

Figure 8. Test 3. Interaction of the shock wave with the coupling interface. Dashed line $\left(t=T_{1}\right):$ the shock wave has not hit the coupling interface. Plain line $\left(t=T_{2}\right):$ the shock wave has passed the coupling interface.
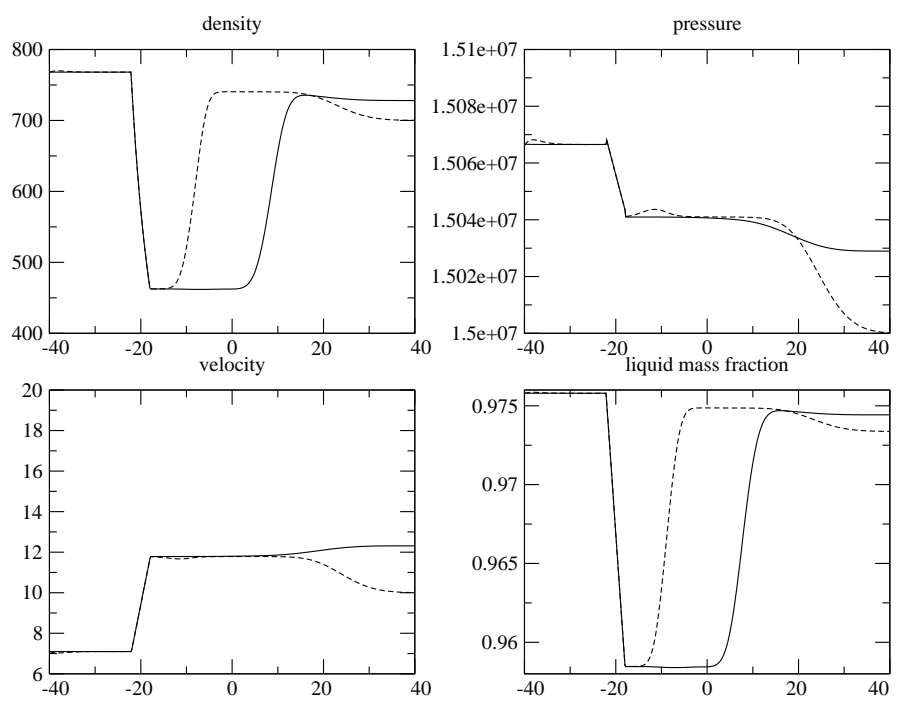

Figure 9. Test 3. Interaction of the contact wave with the coupling interface. Dashed line $\left(t=T_{3}\right):$ the contact wave has not hit the coupling interface. Plain line $\left(t=T_{4}\right):$ the contact wave has passed the coupling interface. 


\section{Conclusion}

In this paper, we study the coupling of two different codes that provide numerical approximations of HEM and HRM models when applying industrial closure laws. It may be seen as a companion work of ${ }^{2}$ in which different coupling techniques are proposed - including the state coupling and the flux coupling approaches - and compared in an idealized thermodynamic frame. Here, we analyze one of these methods when the thermodynamic closures mimick the ones used in industrial codes. The two models HEM and HRM have been recalled, together with closure laws including EOS. We have chosen here a coupling method, that makes use of relaxation techniques. It is essentially different from the one that has been investigated in, ${ }^{26}$ which basically relies on ideas introduced by Greenberg and Le Roux $\left({ }^{21}\right)$. Its simplicity is appealing, and it enables us to circumvent the tough problem of resonance at the coupling interface.

Some properties of both models, and the main properties of algorithms involved in associated codes have been given or recalled. Some properties of the coupling method have also been detailed. One may say that the whole approach is rather efficient, and we would like to emphasize the rather nice behaviour of the coupled approach when contact waves travel through the coupling interface. The reader must be aware that some numerical properties deeply rely on the approximate Godunov schemes $\left({ }^{14}\right)$ which are used in both codes. The coupling approach preserves the conservative form of mass, momentum and total energy equations. It also seems worth emphasizing the following features:

- If one restricts to coarse meshes, which is obviously the correct framework for industrial applications, it also occurs that incompatibilities between formulations may be smoothed with this formulation, owing to the dissipative nature of upwinding techniques which have been applied inside codes and at the coupling interface. This is indeed flagrant if one focuses on relaxation time scales. Some of the numerical results presented $\mathrm{in}^{27}$ confirm that one can cope with decorrelated signals (owing to very large relaxation times in the HRM model) on coarse meshes, but that this no longer holds when refining the mesh. This is much in favour of the present coupling approach, since it may be understood as a tool which automatically checks whether both models on each side of the interface are in agreement or not.

- A much deeper insight on thermodynamics is actually deserved. On the basis of ${ }^{4}$, we know that small discrepancies among EOS may result in rather high differences in results. Special emphasis should be given on the respective saturation curves for the liquid phase and the vapour phase. Some comments may be picked up in this manuscript, which might perhaps help improving the global formulation (see Appendix 4). The reader is also referred to. ${ }^{8,28}$

- In this work, we have focused on compatible closure laws for the HEM and HRM models, and this results in a rather good behaviour in the coupling of both models. We recall that the case of the interfacial coupling of models involving different thermodynamics has been examined in ${ }^{4}$ and. ${ }^{10}$ It clearly suggests that it would be a better idea to use the same thermodynamic tables in both codes.

- Another problem concerns the formulation of mass transfer terms. The one which has been considered here relies on: $\Gamma=K(1-C) C\left(h_{l}^{s}(P)-h_{l}\left(P, \rho_{l}\right)\right)$. Alternative formulations that preserve entropic properties should actually be preferred (see for instance ${ }^{8,9,28}$ ).

- Eventually, we would like to emphasize that the underlying ideas of the present work have been used in order to couple a six-equation two-phase flow model together with the HRM model ( $\left.\operatorname{see}^{24,25}\right)$.

\section{Acknowledgements}

The work presented here has been achieved in the framework of the NEPTUNE project, with financial support from CEA (Commissariat à l'Energie Atomique), EDF (Electricité de France), IRSN (Institut de Radioprotection et de Sûreté Nucléaire) and AREVA-NP. Computational facilities were provided by EDF. The third author has been supported by EDF through a EDF/CIFRE contract during his PhD thesis. Part of the work has also benefited from fruitful discussions with the working group, ${ }^{1}$ and with Thierry Gallouët, who are greatly acknowledged for their help and advise. 


\section{Appendix 1: The VFRoe-ncv scheme and its modified version}

\section{A. The VFRoe-ncv scheme}

We define herein the basic first order approximate Godunov scheme and restrict to regular meshes of size $\Delta x$ such that: $\Delta x=x_{i+\frac{1}{2}}-x_{i-\frac{1}{2}}, i \in \mathbb{Z}$. We denote $\Delta t$ the time step, where $\Delta t=t^{n+1}-t^{n}, n \in \mathbb{N}$. In order to approximate solutions of the exact solution $W \in \mathbb{R}^{p}$ of the conservative hyperbolic system:

$$
\left\{\begin{array}{l}
\partial_{t}(W)+\partial_{x}(F(W))=0 \\
W(x, 0)=W_{0}(x)
\end{array}\right.
$$

with $F(W)$ in $\mathbb{R}^{p}$. Let $W_{i}^{n}$ be the approximate value of $\frac{1}{\Delta x} \int_{x_{i-\frac{1}{2}}}^{x_{i+\frac{1}{2}}} W\left(x, t^{n}\right) d x$. Integrating over $\left[x_{i-\frac{1}{2}} ; x_{i+\frac{1}{2}}\right] \times$ $\left[t^{n} ; t^{n+1}\right]$ provides:

$$
W_{i}^{n+1}=W_{i}^{n}-\frac{\Delta t}{\Delta x}\left(\phi_{i+\frac{1}{2}}^{n}-\phi_{i-\frac{1}{2}}^{n}\right)
$$

The numerical flux $\phi_{i+\frac{1}{2}}^{n}$ through the interface $\left\{x_{i+\frac{1}{2}}\right\} \times\left[t^{n} ; t^{n+1}\right]$ is defined below. The time step must agree with a CFL condition detailed below. The flux $\phi_{i+\frac{1}{2}}^{n}$ depends on $W_{i}^{n}$ and $W_{i+1}^{n}$ when restricting to first order schemes. The approximate Godunov flux $\phi\left(W_{L}, W_{R}\right)$ is obtained by solving exactly the following linear 1D Riemann problem:

$$
\left\{\begin{array}{l}
\partial_{t}(Y)+B(\hat{Y}) \partial_{x}(Y)=0 \\
Y(x, 0)= \begin{cases}Y_{L} & \text { if } x<0 \\
Y_{R} & \text { otherwise }\end{cases}
\end{array}\right.
$$

and initial condition: $Y_{L}=Y\left(W_{i}\right)$ and $Y_{R}=Y\left(W_{i+1}\right)$. The matrix $B$ is defined as:

$$
B(Y)=\left(W_{, Y}(Y)\right)^{-1} A(W(Y)) W_{, Y}(Y)
$$

where $A(W)$ is the Jacobian matrix of flux $F(W))$. Once the exact solution $Y^{*}\left(\frac{x}{t} ; Y_{L}, Y_{R}\right)$ of this approximate linear problem (43) is obtained, the numerical flux is defined as:

$$
\phi\left(W_{L}, W_{R}\right)=F\left(W\left(Y^{*}\left(0 ; Y_{L}, Y_{R}\right)\right)\right)
$$

Let us set $\widetilde{l_{k}}, \widetilde{\lambda_{k}}$ and $\widetilde{r_{k}}, k=1, \ldots, p$, left eigenvectors, eigenvalues and right eigenvectors of matrix $B(\hat{Y})$ respectively. The solution $Y^{*}\left(\frac{x}{t} ; Y_{L}, Y_{R}\right)$ of the linear Riemann problem is defined everywhere (except along $\left.\frac{x}{t}=\widetilde{\lambda_{k}}\right)$ :

$$
\begin{aligned}
Y^{*}\left(\frac{x}{t} ; Y_{L}, Y_{R}\right) & =Y_{L}+\sum_{\frac{x}{t}>\widetilde{\lambda_{k}}}\left({ }^{t} \widetilde{l_{k}} \cdot\left(Y_{R}-Y_{L}\right)\right) \widetilde{r_{k}} \\
& =Y_{R}-\sum_{\frac{x}{t}<\widetilde{\lambda_{k}}}\left({ }^{t} \widetilde{l_{k}} \cdot\left(Y_{R}-Y_{L}\right)\right) \widetilde{r_{k}}
\end{aligned}
$$

The only remaining unknown is the mean $\hat{Y}$ which must comply with the condition:

$$
\hat{Y}\left(Y_{l}=Y_{0}, Y_{r}=Y_{0}\right)=Y_{0}
$$

The standard average which is used is:

$$
\hat{Y}\left(Y_{L}, Y_{R}\right)=\left(Y_{L}+Y_{R}\right) / 2
$$

The explicit form of the approximate Godunov scheme will be:

$$
W_{i}^{n+1}-W_{i}^{n}+\frac{\Delta t}{\Delta x}\left(F\left(W\left(Y^{*}\left(0 ; Y_{i}^{n}, Y_{i+1}^{n}\right)\right)\right)-F\left(W\left(Y^{*}\left(0 ; Y_{i-1}^{n}, Y_{i}^{n}\right)\right)\right)\right)=0
$$


Remark. A different prediction is obtained using instead:

$$
\hat{Y}\left(Y_{L}, Y_{R}\right)=Y^{*}\left(0 ; Y_{L}, Y_{R}\right)
$$

where the approximate value at the interface $Y^{*}\left(0 ; Y_{L}, Y_{R}\right)$ is obtained solving (43) with:

$$
\hat{Y}\left(Y_{L}, Y_{R}\right)=\left(Y_{L}+Y_{R}\right) / 2
$$

This correspond to the WFRoe-ncv scheme.

\section{B. The classical VFRoe-ncv scheme for the interface model}

We present here the VFRoe-ncv scheme for the system (32) using the variable $Z^{\top}=(C, \rho, U, P, Y)$. We denote by $W$ the conservative variable, $W^{\top}=(\rho C, \rho, \rho U, \rho E, \rho Y)$. The Jacobian matrix associated to the flux $F(Z)^{\top}=\left(\rho U C, \rho U, \rho U^{2}+P, U\left(\rho e(P, \rho, C, Y)+\rho U^{2} / 2+P\right), \rho U Y\right)$ is equivalent to $B(Z)$ :

$$
B(Z)=\left(\begin{array}{ccccc}
U & 0 & 0 & 0 & 0 \\
0 & U & \rho & 0 & 0 \\
0 & 0 & U & 1 / \rho & 0 \\
0 & 0 & \rho a^{2} & U & 0 \\
0 & 0 & 0 & 0 & U
\end{array}\right)
$$

where a is defined by the formula (33).

Remark. Owing to the block-diagonal structure of $B(Z)$, it is easy to pick out information about the structure of the Jacobian of the HRM system (4) with $a^{2}$ defined by (17) or the HEM system (22) with $a^{2}$ defined by (29).

The eigenvalues $\lambda_{i}$ and the corresponding right eigenvectors $r_{i}$ are:

$$
\begin{array}{ll}
\lambda_{1}=U-a ; & r_{1}^{\top}=\left(0,1,-a / \rho, a^{2}, 0\right) \\
\lambda_{2}=U ; & r_{2}^{\top}=(1,0,0,0,0) \\
\lambda_{3}=U ; & r_{3}^{\top}=(0,1,0,0,0) \\
\lambda_{4}=U ; & r_{4}^{\top}=(0,0,0,0,1) \\
\lambda_{5}=U+a ; & r_{5}^{\top}=\left(0,1,+a / \rho, a^{2}, 0\right)
\end{array}
$$

The matrix of the right eigenvectors is $\Omega=\left(r_{2}, r_{3}, r_{1}, r_{5}, r_{4}\right)$ and:

$$
\begin{gathered}
\Omega(Z)=\left(\begin{array}{ccccc}
1 & 0 & 0 & 0 & 0 \\
0 & 1 & 1 & 1 & 0 \\
0 & 0 & -a / \rho & a / \rho & 0 \\
0 & 0 & a^{2} & a^{2} & 0 \\
0 & 0 & 0 & 0 & 1
\end{array}\right) \\
\Omega(Z)^{-1}=\frac{-\rho}{2 a^{3}}\left(\begin{array}{ccccc}
-2 a^{3} / \rho & 0 & 0 & 0 & 0 \\
0 & -2 a^{3} / \rho & 0 & 2 a / \rho & 0 \\
0 & 0 & a^{2} & -a / \rho & 0 \\
0 & 0 & -a^{2} & -a / \rho & 0 \\
0 & 0 & 0 & 0 & -2 a^{3} / \rho
\end{array}\right)
\end{gathered}
$$

We define the vector $\alpha^{\top}=\left(\alpha_{2}, \alpha_{3}, \alpha_{1}, \alpha_{5}, \alpha_{4}\right)$ as:

$$
\alpha=\Omega(\bar{Z})^{-1}\left(Z_{R}-Z_{L}\right)
$$

where $Z_{R}$ and $Z_{L}$ are the right and left states of the Riemann problem, and $\bar{Z}=\frac{Z_{R}+Z_{L}}{2}$. The solution of the linearized Riemann problem associated to $J(\bar{Z})$ and the states $\left(Z_{R}, Z_{L}\right)$ is (see figure $(10)$ ),

$$
Z\left(x / t ; Z_{L}, Z_{R}\right)=Z_{L}+\sum_{\bar{\lambda}_{i}<x / t} \alpha_{i} \bar{r}_{i}=Z_{R}-\sum_{\bar{\lambda}_{i}>x / t} \alpha_{i} \bar{r}_{i}
$$

28 of 40 
with:

$$
\bar{\lambda}_{i}=\lambda_{i}(\bar{Z}) \quad \text { and } \quad \bar{r}_{i}=r_{i}(\bar{Z})
$$

This can be written in a slightly different form:

$$
Z\left(x / t ; Z_{L}, Z_{R}\right)=\left\{\begin{array}{l}
Z_{L} \text { if } x / t<\bar{\lambda}_{1} \\
Z_{1} \text { if } \bar{\lambda}_{1}<x / t<\bar{\lambda}_{2} \\
Z_{2} \text { if } \bar{\lambda}_{2}<x / t<\bar{\lambda}_{5} \\
Z_{R} \text { if } \bar{\lambda}_{5}<x / t
\end{array}\right.
$$

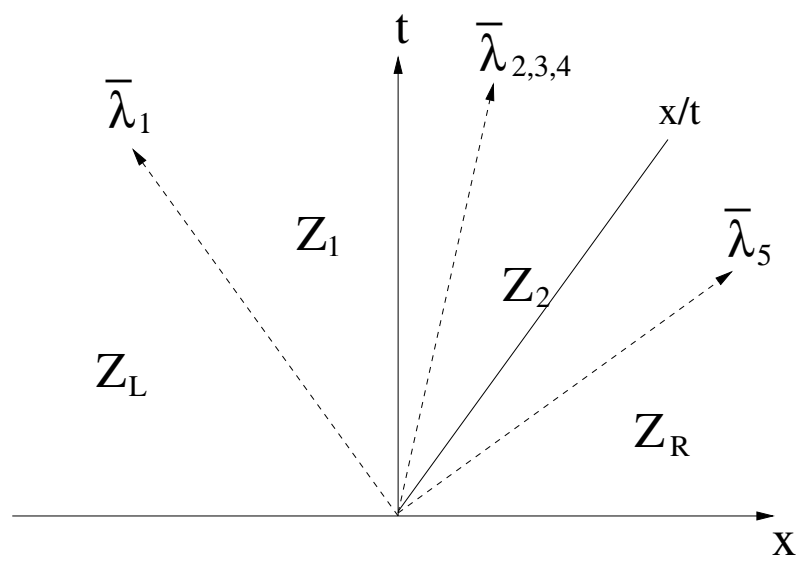

Figure 10. The four different states of the solution of the linearized Riemann problem

We set $\Delta \Phi=\Phi_{R}-\Phi_{L}$. If we apply the formula (44) we find that the two intermediate states are:

$$
Z_{1}=\left(\begin{array}{c}
C_{L} \\
\rho_{L}+\frac{\Delta P}{2 \bar{a}^{2}}-\frac{\bar{\rho} \Delta U}{2 \bar{a}} \\
\bar{U}-\frac{\Delta P}{2 \bar{\rho} \bar{a}} \\
\bar{P}-\bar{\rho} \bar{a} \frac{\Delta U}{2} \\
Y_{L}
\end{array}\right) \quad Z_{2}=\left(\begin{array}{c}
C_{R} \\
\rho_{R}-\frac{\Delta P}{2 \bar{a}^{2}}-\frac{\bar{\rho} \Delta U}{2 \bar{a}} \\
\bar{U}-\frac{\Delta P}{2 \bar{\rho} \bar{a}} \\
\bar{P}-\bar{\rho} \bar{a} \frac{\Delta U}{2} \\
Y_{R}
\end{array}\right)
$$

In the above formulas $\bar{a}$ is the sound speed calculated at the average state $\bar{Z}$, that is $\bar{a}=a(\bar{Z})$. The numerical flux for the classical VFRoe-ncv scheme is computed using $Z^{*}=Z\left(x / t=0 ; Z_{L}, Z_{R}\right)$ (for example in the configuration of the figure (10) $Z^{*}=Z_{1}$ ).

Remark. - The classical VFRoe-ncv scheme using variables $(\rho, U, P)$ for the HEM model (22):

The linear Riemann Problem associated to the system (22) has two intermediate states as shown in figure (10). The intermediate states are:

$$
Z_{1}=\left(\begin{array}{c}
\rho_{L}+\frac{\Delta P}{2 \bar{a}_{H E M}^{2}}-\frac{\bar{\rho} \Delta U}{2 \bar{a}_{H E M}} \\
\bar{U}-\frac{\Delta}{2 \overline{\bar{\rho}} \overline{\bar{a}}_{H E M}} \\
\bar{P}-\bar{\rho} \bar{a} \bar{a}_{H E M} \frac{\Delta U}{2}
\end{array}\right) \quad Z_{2}=\left(\begin{array}{c}
\rho_{R}-\frac{\Delta P}{2 \bar{a}_{H E M}^{2}}-\frac{\bar{\rho} \Delta U}{2 \bar{a}_{H E M}} \\
\bar{U}-\frac{\Delta P}{2 \overline{\bar{a}} \overline{\bar{a}}_{H E M}} \\
\bar{P}-\bar{\rho} \overline{a_{H E M}} \frac{\Delta U}{2}
\end{array}\right)
$$

- The classical VFRoe-ncv scheme using variables $(C, \rho, U, P)$ for the HRM model (4): The linear Riemann Problem associated to the system (4) has two intermediate states as shown in figure (10). The intermediate states are:

$$
Z_{1}=\left(\begin{array}{c}
C_{L} \\
\rho_{L}+\frac{\Delta P}{2 \bar{a}_{H R M}^{2}}-\frac{\bar{\rho} \Delta U}{2 \overline{2} \bar{a}_{H R M}} \\
\bar{U}-\frac{\Delta \overline{\bar{a}_{H}}}{2 \overline{\bar{a}} \bar{a}_{H M}} \\
\bar{P}-\bar{\rho} \bar{a} H R M \frac{\Delta U}{2}
\end{array}\right) \quad Z_{2}=\left(\begin{array}{c}
C_{R} \\
\rho_{R}-\frac{\Delta P}{2 \bar{a}_{H R M}^{2}}-\frac{\bar{\rho} \Delta U}{2 \bar{a}_{H R M}} \\
\bar{U}-\frac{\Delta P}{2 \bar{\rho} \bar{a}_{H R M}} \\
\bar{P}-\bar{\rho} \bar{a}_{H R M} \frac{\Delta U}{2}
\end{array}\right)
$$

29 of 40 


\section{A modified VFRoe-ncv scheme for the interface model}

This modified VFRoe-ncv scheme for the interface model differs from the classical VFRoe-ncv scheme in the choice of the average state which is not a classical average. We use here the "average state":

$$
\tilde{Z}^{\top}=(\bar{C}, \bar{\rho}, \bar{U}, \bar{P}, \tilde{Y}) ; \quad \tilde{Y}=\left\{\begin{array}{l}
0 \text { if } \bar{U}>0 \\
1 \text { if } \bar{U}<0
\end{array}\right.
$$

This implies that the sound speed $\bar{a}=a(\bar{P}, \bar{\rho}, \bar{C}, \bar{Y})$ is replaced by,

$$
\tilde{a}=\left\{\begin{array}{l}
a_{H E M}(\bar{P}, \bar{\rho}) \text { if } \bar{U}>0 \\
a_{H R M}(\bar{P}, \bar{\rho}, \bar{C}) \text { if } \bar{U}<0
\end{array}\right.
$$

This provides a new solution $Z^{\#}$ (note that $Y^{\#}=\tilde{Y}$ ). If we then explicit the numerical flux we find:

$$
F\left(Z^{\#}\right)^{\top}=\left((\rho U C)^{\#},(\rho U)^{\#},\left(\rho U^{2}+P\right)^{\#},\left(U\left(\rho e(P, \rho, C, Y)+\rho U^{2} / 2+P\right)\right)^{\#},(\rho U Y)^{\#}\right)
$$

with,

$$
e^{\#}=\left\{\begin{array}{l}
e_{H E M}\left(P^{\#}, \rho^{\#}\right) \text { if } \bar{U}>0 \\
e_{H R M}\left(P^{\#}, \rho^{\#}, C^{\#}\right) \text { if } \bar{U}<0
\end{array}\right.
$$

Eventually, if $\bar{U}>0$, this is equivalent to apply the VFRoe-ncv scheme to the HEM system (31) with in addition the equations on $Y$ and $C$ of (32); if $\bar{U}<0$, this is equivalent to apply the VFRoe-ncv scheme to the HRM system (4) with in addition the equation on $Y$ of (32). 


\section{Appendix 2: Computation of $C^{n+1}$ and $P^{n+1}$ for the finite time relaxation step}

In order to compute the finite relaxation step we need to explain how $C^{n+1}$ and $P^{n+1}$ are updated. They are the solution at the time $\Delta t$ of the system (ODE) composed by the first and fourth equations of (40) with the initial condition: $C(t=0)=C^{\hat{n}}$ and $P(t=0)=P^{\hat{n}}$.

The main time step $\Delta t$ is cut into $N$ small uniform time steps $\delta t=\Delta t / N$. In the following $C_{i}$ and $P_{i}$ will stand for the successive approximations of $C$ and $P$ at the successive times $t=i \times \delta t$. Thus we will have:

$$
\begin{aligned}
C_{0} & :=C^{\hat{n}} \text { and } P_{0}:=P^{\hat{n}} \\
C^{n+1} & :=C_{N} \text { and } P^{n+1}:=P_{N}
\end{aligned}
$$

The chain rule which provides $\left(C_{i+1}, P_{i+1}\right)$ in terms of $\left(C_{i}, P_{i}\right)$ is the following:

- $C_{i+1}$ is the exact solution at the time $t=\delta t$ of the ODE system:

$$
\left\{\begin{array}{l}
\frac{d}{d t} C(t)=\Gamma\left(P_{i}, \rho^{\hat{n}}, C(t)\right) \\
C(t=0)=C_{i}
\end{array}\right.
$$

where we have,

$$
\Gamma\left(P_{i}, \rho^{\hat{n}}, C(t)\right)=(1-C(t))\left(C_{e q}\left(P_{i}, \rho^{\hat{n}}\right)-C(t)\right) \frac{1}{\Theta\left(P_{i}, \rho^{\hat{n}}, \tau_{0}\right)}
$$

with the time scale,

$$
\Theta\left(P, \rho, \tau_{0}\right)=\frac{\tau_{0}}{G_{2}(P, \rho)}
$$

where $G_{2}$ defined by equation (18). We impose that the numerical approximation $C_{i+1}$ respects the relations:

$$
\begin{gathered}
\left(C_{i+1}-C_{e q}\left(P_{i}, \rho^{\hat{n}}\right)\right)\left(C_{i}-C_{e q}\left(P_{i}, \rho^{\hat{n}}\right)\right)>0 \\
\left(1-C_{i+1}\right)\left(1-C_{i}\right)>0
\end{gathered}
$$

This relation corresponds to the fact that the analytical solution $C(t)$ of $(45)$ can not cross the poles $C=C_{e q}\left(P_{i}, \rho^{\hat{n}}\right)$ and $C=1$.

We get $C_{i+1}=C_{i}$ if $C_{i}=1$ and if $C_{i} \neq 1$,

$$
C_{i+1}=\frac{b_{i+1}+C_{e q}\left(P_{i}, \rho^{\hat{n}}\right)}{1+b_{i+1}}
$$

with:

$$
\begin{gathered}
b_{i+1}=\frac{C_{i}-C_{e q}\left(P_{i}, \rho^{\hat{n}}\right)}{1-C_{i}} e^{\left(a_{i+1} \delta t\right)} \\
a_{i+1}=-\left(1-C_{e q}\left(P_{i}, \rho^{\hat{n}}\right)\right) \frac{G_{2}\left(P_{i}, \rho^{\hat{n}}\right)}{\tau_{0}}
\end{gathered}
$$

- To compute $P_{i+1}$, we use the fourth equation of the system (40) in the form

$$
\partial_{t}(P)=-\left(\partial_{P}\left(e_{H R M}\right)\right)^{-1} \partial_{C}\left(e_{H R M}\right) \partial_{t}(C)
$$

and we make a rough integration:

$$
P_{i+1}=P_{i}+\delta t\left(\partial_{t}(P)\right)\left(P_{i}, \rho^{\hat{n}}, C_{i+1}, Y^{\hat{n}}\right)
$$

that is:

$$
P_{i+1}=P_{i}-\left(C_{i+1}-C_{i}\right)\left(\frac{\partial_{C}\left(e_{H R M}\right)}{\partial_{P}\left(e_{H R M}\right)}\right)\left(P_{i}, \rho^{\hat{n}}, C_{i+1}, Y^{\hat{n}}\right)
$$

31 of 40 
A numerical maximum principle :

We assume that $C_{e q}\left(P_{i}, \rho^{\hat{n}}\right)$ remains in $[0,1]$.

- We assume that $0 \leq C_{e q}\left(P_{i}, \rho^{\hat{n}}\right) \leq C_{i} \leq 1$ :

This implies that $b_{i+1} \geq 0$. Hence using the equation (49) we get:

$$
0 \leq C_{i+1} \leq 1
$$

- We assume that $0 \leq C_{i} \leq C_{e q}\left(P_{i}, \rho^{\hat{n}}\right) \leq 1$ :

This implies that $b_{i+1} \leq 0$. We set,

$$
H(b)=\frac{b+C_{e q}\left(P_{i}, \rho^{\hat{n}}\right)}{1+b} \quad \text { and } \quad J(b)=1-H(b)
$$

Thus we have $H\left(b_{i+1}\right)=C_{i+1}$ and $J\left(b_{i+1}\right)=1-C_{i+1}$. When we examine the positivity of both $H(b)$ and $J(b)$ we find that it is equivalent to the relation $b \geq-C_{e q}\left(P_{i}, \rho^{\hat{n}}\right)$. This leads to the condition on the time step $\delta t$ :

$$
\delta t \geq 0
$$

which is obviously fulfilled.

Hence we get for $\delta t \geq 0$ :

$$
0 \leq C_{i+1} \leq 1
$$

We thus have the following property.

\section{Property:}

The following maximum principle holds for the finite time relaxation step. If we assume that:

(i) the time step $\delta t$ is positive,

(ii) the pole $C_{e q}\left(P_{i}, \rho^{\hat{n}}\right)$ of the ODE system (45) remains in $[0,1]$,

(iii) $0 \leq C_{i} \leq 1$

Then we have $0 \leq C_{i+1} \leq 1$.

Remark. Actually, the only assumption is that $C_{e q}\left(P_{i}, \rho^{\hat{n}}\right)$ remains in $[0,1]$. The finite time relaxation step does not ensure that $C_{e q}\left(P_{i+1}, \rho^{\hat{n}}\right)$ will remain in $[0,1]$ (see appendix 4 for similar comments on $C_{e q}$ ). 


\section{Appendix 3: On the discrete preservation of contact waves in HEM and HRM models}

\section{A. HRM}

This paragraph is strongly connected with the section dealing with the numerical schemes. It refers to the work exposed in. ${ }^{14}$ We formally set $\Gamma=0$ in the system (4). A pure contact solution is an analytical solution of (4) (with $\Gamma=0$ ) of the form:

$$
\left\{\begin{array}{l}
C(x, t)=C_{0}\left(x-U_{0} t\right) \\
\rho(x, t)=\rho_{0}\left(x-U_{0} t\right) \\
U(x, t)=U_{0} \\
P(x, t)=P_{0}
\end{array}\right.
$$

When the initial condition is such that the pressure and the velocity are uniform, the initial profiles of $C$ and density (i.e. $C_{0}(x)$ and $\left.\rho_{0}(x)\right)$ are advected with speed $U_{0}$. We say that the contact solution is preserved by the conservative numerical scheme if the numerical approximations of the velocity and the pressure remain constant in each cell of the discrete domain.

According to ${ }^{14}$ the discrete contact solution is respected if two conditions are fulfilled:

(i) The internal energy must be of the form:

$$
\rho e=f_{1}(P)+\rho f_{2}(P)+\rho C f_{3}(P)
$$

(ii) The numerical flux involved in the conservative scheme preserves the contact solution.

Remark. The second point (ii) is achieved when using either the Godunov scheme or VFRoe-ncv scheme with variables $(C, \rho, U, P)$.

The two conditions are necessary to preserve the discrete profiles of both $U$ and $P$. If (i) is not fulfilled, the contact solution will not be respected whatever the conservative numerical scheme is (even for the Godunov scheme). We insist that whatever the continuous function defining the EOS (i.e. $\left.(P, \rho, C) \mapsto e_{H R M}(P, \rho, C)\right)$ for the HRM model is, the vector (52) is an analytical solution of the system (4) without any source term.

Rewriting (12) we find that the internal energy is of the form:

$$
\rho e=\phi_{1}(P)+\rho \phi_{2}(P)+\rho C\left(-\phi_{2}(P)\right)
$$

with:

$$
\begin{gathered}
\phi_{1}(P)=\left(\delta_{l}-1\right) P \\
\phi_{2}(P)=\left[\left(1-\frac{\delta_{l}}{\delta_{v}}\right) h_{v}^{s}(P)\right]
\end{gathered}
$$

Hence this internal energy allows the discrete preservation of the contact wave since the condition (i) is fulfilled. In our numerical tests the convective part of the HRM model will be computed using a VFRoe-ncv scheme with variables $(C, \rho, U, P)$ that fulfills the condition (ii) $\left(\mathrm{see}^{14}\right)$.

\section{B. HEM}

The internal energy of the HEM model takes the form:

$$
\rho e=\phi_{4}(P)+\rho \phi_{5}(P)
$$

with:

- In the pure liquid domain $D_{l}$ :

$$
\phi_{4}(P)=\left(\delta_{l}-1\right) P \quad \text { and } \quad \phi_{5}(P)=0
$$


- In the two-phase domain $D_{2 \Phi}$ :

$$
\begin{gathered}
\phi_{4}(P)=\left[-P+\frac{h_{v}^{s}(P)-h_{l}^{s}(P)}{\tau_{v}^{s}(P)-\tau_{l}^{s}(P)}\right] \\
\phi_{5}(P)=\left[\frac{\tau_{v}^{s}(P) h_{l}^{s}(P)-\tau_{l}^{s}(P) h_{v}^{s}(P)}{\tau_{v}^{s}(P)-\tau_{l}^{s}(P)}\right]
\end{gathered}
$$

Following, ${ }^{14}$ we conclude that this EOS allows the preservation of the discrete contact solution in the pure HEM domain. Hence when the discrete contact solution evolves in a single domain (i.e. in the pure liquid or in the two-phase domain), it is preserved provided that the numerical scheme preserves the contact solution. This is no longer true when going through the boundary between the domains $D_{2 \Phi}$ and $D_{l}$ (a numerical example is available $\mathrm{in}^{27}$ ). Once again, we recall that the convective part of the HEM model is computed using a VFRoe-ncv scheme with variables $(\rho, U, P)$. Hence, the condition (ii) is guaranteed $\left(\right.$ see $\left.^{14}\right)$.

\section{Contact waves travelling through the coupling interface}

We know that the discrete contact solutions are preserved by the HEM and the HRM models. We examine herein what happens when a contact wave hits the coupling interface (as described by figure (11)).

HEM

HRM

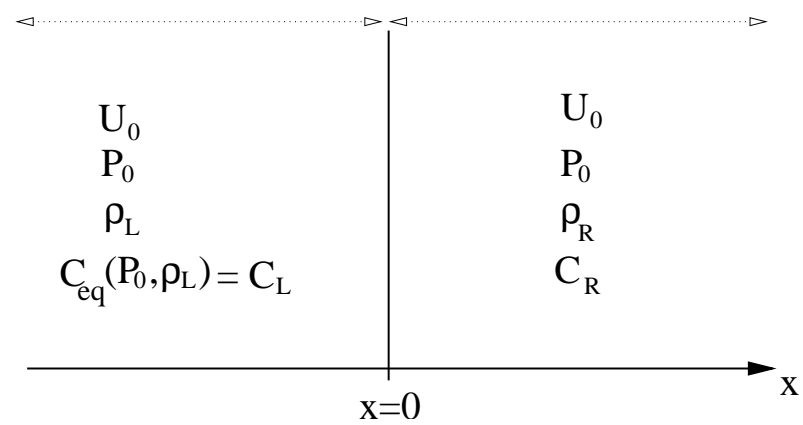

Figure 11. Initial data for a contact test case hitting the coupling interface

Remark. Thanks to the preservation of the contact wave for sole HEM and HRM models, we only need to examine the discrete values of the variables in the two cells on each side of the coupling interface.

a ) $\underline{U_{0}>0}$

A problem may only occur in the first cell in the HRM domain. After one time step of our coupling scheme involving VFRoe-ncv scheme and described above in appendix 1, we get in the first cell of the HRM domain the values $(\rho, U, P, C)$ defined by:

$$
\begin{aligned}
\rho-\rho_{R}+\lambda\left(\rho_{R} U_{0}-\rho_{L} U_{0}\right) & =0 \\
\rho U-\rho_{R} U_{0}+\lambda\left(\rho_{R} U_{0}^{2}+P_{0}-\rho_{L} U_{0}^{2}-P_{0}\right) & =0 \\
\rho E_{H R M}-\rho_{R} E_{H R M}^{R}+\lambda\left(U_{0}\left(\rho_{R} E_{H R M}^{R}+P_{0}\right)-U_{0}\left(\rho_{L} E_{H E M}^{L}+P_{0}\right)\right) & =0 \\
\rho C-\rho_{R} C_{R}+\lambda\left(\rho_{R} U_{0} C_{R}-\rho_{L} U_{0} C_{e q}^{L}\right) & =0
\end{aligned}
$$

with $\lambda=\frac{\Delta t}{\Delta x}$ and:

$$
\begin{gathered}
E_{H R M}=e_{H R M}(P, \rho, C)+\frac{U^{2}}{2} \\
E_{H R M}^{R}=e_{H R M}\left(P_{0}, \rho_{R}, C_{R}\right)+\frac{U_{0}^{2}}{2}
\end{gathered}
$$

34 of 40 


$$
\begin{gathered}
E_{H E M}^{L}=e_{H E M}\left(P_{0}, \rho_{L}\right)+\frac{U_{0}^{2}}{2}=e_{H R M}\left(P_{0}, \rho_{L}, C_{e q}^{L}\right)+\frac{U_{0}^{2}}{2} \\
C_{e q}^{L}=C_{e q}\left(P_{0}, \rho_{L}\right)
\end{gathered}
$$

Using the internal energy in the form (54), the system (56)-(59) provides:

$$
\begin{array}{r}
\rho-\rho_{R}+\lambda U_{0}\left(\rho_{R}-\rho_{L}\right)=0 \\
U=U_{0} \\
\left(\Phi_{1}(P)-\Phi_{1}\left(P_{0}\right)\right)+\rho(1-C)\left(\Phi_{2}(P)-\Phi_{2}\left(P_{0}\right)\right)=0 \\
\rho C-\rho_{R} C_{R}+\lambda\left(\rho_{R} U_{0} C_{R}-\rho_{L} U_{0} C_{e q}^{L}\right)=0
\end{array}
$$

The equation (62) implies that for any $\rho \geq 0$ and for any $\rho(1-C) \geq 0$ defined by (60) and (63):

$$
\Phi_{1}(P)=\Phi_{1}\left(P_{0}\right) \quad \text { and } \quad \Phi_{2}(P)=\Phi_{2}\left(P_{0}\right)
$$

and thanks to the bijectivity of the applications $P \mapsto \Phi_{1}(P)$ and $P \mapsto \Phi_{2}(P)$ this leads to $P=P_{0}$.

Thus the contact wave passing from the HEM model to the HRM model will be preserved.

b ) $\underline{U_{0}<0}$

A problem may only occur in the first cell in the HEM domain. One time step of the coupling described above provides in the first cell of the HEM domain the values $(\rho, U, P)$ defined by:

$$
\begin{aligned}
\rho-\rho_{L}+\lambda\left(\rho_{R} U_{0}-\rho_{L} U_{0}\right) & =0 \\
\rho U-\rho_{L} U_{0}+\lambda\left(\rho_{R} U_{0}^{2}+P_{0}-\rho_{L} U_{0}^{2}-P_{0}\right) & =0 \\
\rho E_{H E M}-\rho_{L} E_{H E M}^{L}+\lambda\left(U_{0}\left(\rho_{R} E_{H R M}^{R}+P_{0}\right)-U_{0}\left(\rho_{L} E_{H E M}^{L}+P_{0}\right)\right) & =0
\end{aligned}
$$

with $\lambda=\frac{\Delta t}{\Delta x}$ and:

$$
\begin{gathered}
E_{H E M}=e_{H E M}(P, \rho)+\frac{U^{2}}{2} \\
E_{H R M}^{R}=e_{H R M}\left(P_{0}, \rho_{R}, C_{R}\right)+\frac{U_{0}^{2}}{2} \\
E_{H E M}^{L}=e_{H E M}\left(P_{0}, \rho_{L}\right)+\frac{U_{0}^{2}}{2}
\end{gathered}
$$

Using the internal energy in the form (55), the system (64)-(66) provides:

$$
\begin{array}{r}
\rho-\rho_{L}+\lambda\left(\rho_{R} U_{0}-\rho_{L} U_{0}\right)=0 \\
U=U_{0} \\
\Phi_{4}(P)-\Phi_{4}\left(P_{0}\right)+\rho\left(\Phi_{5}(P)-\Phi_{5}\left(P_{0}\right)\right)+\lambda U_{0} \rho_{R}\left(e_{H R M}^{R}-e_{H E M}^{R}\right)=0
\end{array}
$$

Hence if $P=P_{0}$, the equation (69) reads:

$$
e_{H R M}\left(P_{0}, \rho_{R}, C_{R}\right)=e_{H R M}\left(P_{0}, \rho_{R}, C_{e q}^{R}\right)
$$

which thanks to the bijectivity of $C \mapsto e_{H R M}(P, \rho, C)$ implies that $C_{R}=C_{e q}^{R}$. Then a necessary condition for the respect of the discrete contact solution is:

$$
C_{R}=C_{e q}\left(P_{0}, \rho_{R}\right)
$$

Let us now assume that $C_{R}=C_{e q}\left(P_{0}, \rho_{R}\right)$. Thus $e_{H R M}\left(P_{0}, \rho_{R}, C_{R}\right)=e_{H R M}\left(P_{0}, \rho_{R}, C_{e q}^{R}\right)$, and the pressure $P$ is a solution of the equation:

$$
\Phi_{4}(P)-\Phi_{4}\left(P_{0}\right)+\rho\left(\Phi_{5}(P)-\Phi_{5}\left(P_{0}\right)\right)=0
$$


where $\rho$ is defined by the equation (67). The preceding equation must be fulfilled for any $\rho$. Thus it implies that

$$
\Phi_{4}(P)=\Phi_{4}\left(P_{0}\right) \quad \text { and } \quad \Phi_{5}(P)=\Phi_{5}\left(P_{0}\right)
$$

The two equations of (71) can be rewritten as second order polynomials with respect to the variable $P$. Obviously $P_{0}$ is one solution (hence the discriminants of the polynomials are non-negative). It can be shown that the second solution of the second equation of (71) is greater than $P_{M A X}$ for all $P_{0}$ in $\left[P_{M I N}, P_{M A X}\right]$. Thus the second equation of (71) has a unique admissible solution $P=P_{0}$. This implies that $P=P_{0}$ is the unique solution of the system (71). We can deduce that $C_{R}=C_{e q}\left(P_{0}, \rho_{R}\right)$ is a sufficient condition for the preservation of the contact wave.

Finally, the condition $C_{R}=C_{e q}\left(P_{0}, \rho_{R}\right)$ is a necessary and sufficient condition to fulfill for the preservation of the discrete contact wave when hitting the coupling interface.

\section{A contact wave solution of the HRM model which accounts for the source term}

We provide here some simple analytical solution of the HRM model when the source term is present and when the EOS is complex. For a given uniform velocity $U_{0}$, this one is:

- For $x<U_{0} t$ :

$$
\left\{\begin{array}{l}
\rho(x, t)=\rho_{L} \\
C(x, t)=C_{L}(t) \\
U(x, t)=U_{0} \\
P(x, t)=P_{0}(t)
\end{array}\right.
$$

- For $x>U_{0} t$ :

$$
\left\{\begin{array}{l}
\rho(x, t)=\rho_{R} \\
C(x, t)=C_{R}(t) \\
U(x, t)=U_{0} \\
P(x, t)=P_{0}(t)
\end{array}\right.
$$

where $\rho_{L}, \rho_{R}$ are some positive constants and $C_{L}(t), C_{R}(t)$ and $P_{0}(t)$ should comply with :

$$
\left\{\begin{aligned}
\partial_{t}\left(P_{0}\right) & =-\left(\frac{\partial_{C}\left(e_{H R M}\right)}{\partial_{P}\left(e_{H R M}\right)} \Gamma\right)\left(\rho_{L}, P_{0}(t), C_{L}(t)\right) \\
& =-\left(\frac{\partial_{C}\left(e_{H R M}\right)}{\partial_{P}\left(e_{H R M}\right)} \Gamma\right)\left(\rho_{R}, P_{0}(t), C_{R}(t)\right) \\
\partial_{t}\left(C_{L}\right) & =\Gamma\left(\rho_{L}, P_{0}(t), C_{L}(t)\right) \\
\partial_{t}\left(C_{R}\right) & =\Gamma\left(\rho_{R}, P_{0}(t), C_{R}(t)\right)
\end{aligned}\right.
$$

The stiff constraint which occurs in the governing equation of $P_{0}(t)$ guarantees that jump conditions are fulfilled through the characteristic $x / t=U_{0}$. This one becomes trivial when $\partial_{C}\left(e_{H R M}\right)=0$. In our case, where $\partial_{C}\left(e_{H R M}\right)$ is non-zero, some exact solutions can be found when:

$$
\Gamma(\rho, P, C)=\frac{\partial_{P}\left(e_{H R M}\right)}{\partial_{C}\left(e_{H R M}\right)} \quad \Gamma_{0}(P)
$$




\section{Appendix 4: Some results for the HRM model}

\section{A. Positivity results}

We begin by recalling a classical lemma (see ${ }^{16,29}$ for instance).

For any $\Psi(x, t)$, we introduce the decomposition $\Psi(x, t)=\Psi^{+}(x, t)-\Psi^{-}(x, t)$, so that both $0 \leq \Psi^{-}(x, t)$ and $0 \leq \Psi^{+}(x, t)$.

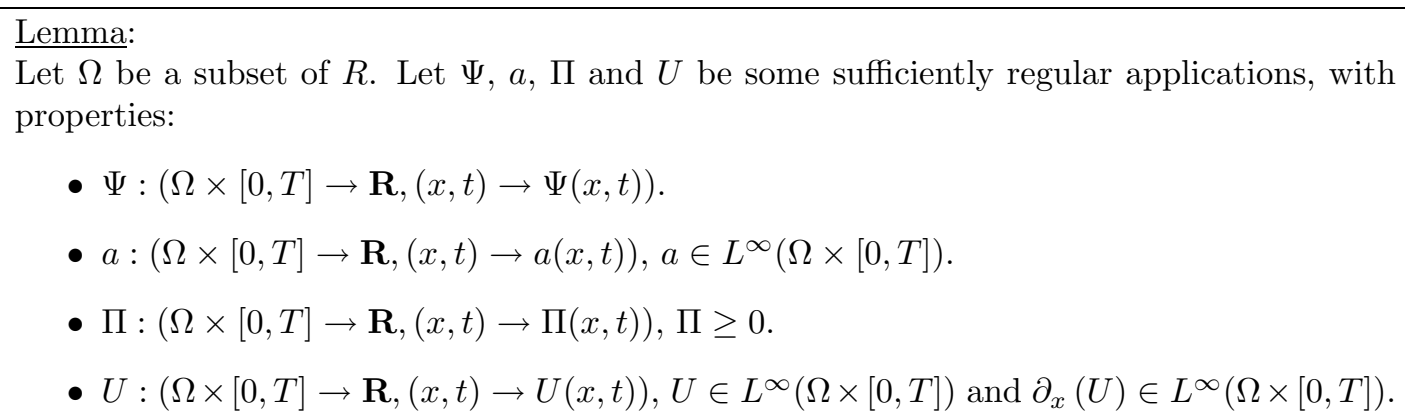

and such that:

$$
\partial_{t}(\Psi(x, t))+U \partial_{x}(\Psi(x, t))=a(x, t) \Psi(x, t)+\Pi(x, t)
$$

Suppose that on $\partial \Omega$, the boundary of $\Omega, \Psi^{-}(x, t)=0$ if $(U \cdot n)(x, t) \leq 0$, where $n$ is the outward normal of $\Omega$. With all these assumptions, if $\Psi(x, t=0) \geq 0$ then for all $0 \leq t \leq T$, $\Psi(x, t) \geq 0$.

In the following we will assume that:

$$
U \in L^{\infty}(\Omega \times[0, T]) \text { and } \partial_{x}(U) \in L^{\infty}(\Omega \times[0, T])
$$

a) Evolution of the density $\rho$ :

The lemma applies to the second equation of (15). It ensures the positivity of the total density $\rho$ :

$$
\rho \geq 0
$$

b) Evolution of the pressure $P$ :

b.1) The fourth equation of (15) can take the form:

$$
\partial_{t}(P)+U \partial_{x}(P)+\hat{\gamma} P \partial_{x}(U)=P J(P, \rho, C)
$$

where the term $J(P, \rho, C)$ is bounded if $\rho$ is bounded and if $\rho(1-C)$ remains positive. The lemma thus applies if $\hat{\gamma} \in L^{\infty}(\Omega \times[0, T])$, and we get:

$$
P>0 \text { if } \rho \text { is bounded and } \rho(1-C)>0
$$

b.2) The fourth equation of (15) does not ensure that $P$ remains in $\left[P_{M I N}, P_{M A X}\right]$. This equation can be written:

$$
\partial_{t}(P)+U \partial_{x}(P)+\hat{\gamma} P \partial_{x}(U)=(1-C)(\bar{C}-C) F(P, \rho)
$$

where $F$ is positive for $P \in\left[P_{M I N}, P_{M A X}\right]$ and $\rho>0$. Hence if we choose $P(x, t=0)=P_{M I N}, U(x, t=$ $0)=U_{0}, \rho(x, t=0)=\rho_{0}$ and $C(x, t=0)=C_{0}$ with the relation $\bar{C}\left(P_{0}, \rho_{0}\right)<C_{0}<1$, then:

$$
\partial_{t}(P)_{\mid t=0}<0
$$

Thus, for $0<\epsilon \leq \epsilon_{0}$, where $\epsilon_{0}>0$ is small enough, we get: $P(x, t=\epsilon)<P_{M I N}$. We may also choose initial conditions such that $P(x, t=\epsilon)>P_{M A X}$. In fact, this makes sense since the only physically relevant lower 
and upper bounds for the pressure are $P_{M I N}=0$, and $P_{M A X}=P_{\text {critical }}$. As discussed above, this particular value of the lower bound will not lead to any problem. The problem is actually less obvious with the upper bound since our governing equations probably no longer make sense around the critical point.

c) Evolution of the liquid mass fraction $C$ :

We make the assumption that $\rho$ is bounded and that $P \in\left[P_{M I N}, P_{M A X}\right]$. It implies that $G_{2}$ is bounded. We set $\Psi_{1}=1-C$. Then the first equation of the system (15), gives:

$$
\partial_{t}\left(\Psi_{1}\right)+U \partial_{x}\left(\Psi_{1}\right)=-\Psi_{1}(x, t)(\bar{C}-C) \frac{G_{2}(P, \rho)}{\tau_{0}}
$$

By applying the lemma mentioned above we find that $C<1$.

We set now $\Psi_{2}=C$. Then the first equation of the system (15), gives:

$$
\partial_{t}\left(\Psi_{2}\right)+U \partial_{x}\left(\Psi_{2}\right)=\Psi_{2}(x, t)(C-1) \frac{G_{2}(P, \rho)}{\tau_{0}}+\Pi
$$

with $\Pi=\bar{C}(1-C) \frac{G_{2}(P, \rho)}{\tau_{0}}$. If we make the assumption that $\bar{C}>0$, the source term $\Pi$ is always positive. By applying the lemma we find that $C>0$.

$$
\begin{aligned}
& C<1 \text { if } \rho \text { is bounded and } P \in\left[P_{M I N}, P_{M A X}\right] \\
& C>0 \text { if } \rho \text { is bounded, } P \in\left[P_{M I N}, P_{M A X}\right] \text { and } \bar{C}>0
\end{aligned}
$$

d) Evolution of the equilibrium liquid mass fraction $\bar{C}$ :

Owing to the latter result, we wonder whether $\bar{C}$ remains in $[0,1]$. We have the equivalence:

$$
0<\bar{C}<1 \Longleftrightarrow \tau_{l}^{s}(P)<\tau<\tau_{v}^{s}(P)
$$

We set $\Psi_{3}=\tau_{v}^{s}(P)-\tau$ and $\Psi_{4}=\tau-\tau_{l}^{s}(P)$. The governing equations for $\Psi_{3}$ and $\Psi_{4}$ are:

$$
\begin{gathered}
\partial_{t}\left(\Psi_{3}\right)+U \partial_{x}\left(\Psi_{3}\right)-\Psi_{3} \partial_{x}(U)+\left(\hat{\gamma} P \frac{d}{d P} \tau_{v}^{s}(P)+\tau_{v}^{s}(P)\right) \partial_{x}(U)=-\frac{\partial_{C}\left(e_{H R M}\right)}{\partial_{P}\left(e_{H R M}\right)} \Gamma \frac{d}{d P} \tau_{v}^{s}(P) \\
\partial_{t}\left(\Psi_{4}\right)+U \partial_{x}\left(\Psi_{4}\right)-\Psi_{4} \partial_{x}(U)-\left(\hat{\gamma} P \frac{d}{d P} \tau_{l}^{s}(P)+\tau_{l}^{s}(P)\right) \partial_{x}(U)=\frac{\partial_{C}\left(e_{H R M}\right)}{\partial_{P}\left(e_{H R M}\right)} \Gamma \frac{d}{d P} \tau_{l}^{s}(P)
\end{gathered}
$$

Hence the lemma cannot be applied. Moreover we can choose initial data such that $\bar{C}>1$ or $\bar{C}<0$. For example with $P(x, t=0)=P_{0}, U(x, t=0)=U_{0}, \rho(x, t=0)=1 / \tau_{v}^{s}\left(P_{0}\right)$ and $C(x, t=0)=C_{0}$ with $\left.C_{0} \in\right] \bar{C}\left(P_{0}, \rho_{0}\right), 1[$ the equation (76) gives:

$$
\partial_{t}\left(\Psi_{3}\right)_{\mid t=0}<0
$$

with $\Psi_{3 \mid t=0}=0$. Then $\Psi_{3 \mid t=0^{+}}<0$ and $\bar{C}_{\mid t=0^{+}}<0$. We may also choose initial conditions such that $\bar{C}_{\mid t=0^{+}}>1$.

Even when the products $\partial_{C}\left(e_{H R M}\right) \Gamma \frac{d}{d P} \tau_{v, l}^{s}(P)$ are null, we may wonder in which case $\bar{C}$ may remain in $[0,1]$. Actually, a sufficient condition would be that :

$$
\left\{\begin{array}{l}
\hat{\gamma} P \frac{d}{d P} \tau_{v}^{s}(P)+\tau_{v}^{s}(P)=A\left(\tau_{v}^{s}(P)-\tau\right) \\
\hat{\gamma} P \frac{d}{d P} \tau_{l}^{s}(P)+\tau_{l}^{s}(P)=B\left(\tau-\tau_{l}^{s}(P)\right)
\end{array}\right.
$$

where $A, B$ should be bounded. The initial guess $(A, B)=(0,0)$ seems unlikely to be a physical solution, since it would imply that $\partial_{P}\left(\frac{\tau_{v}^{s}(P)}{\tau_{v}^{s}(P)}\right)=0$, which is not guaranteed in our case for instance.

We refer to the work of Jaouen $\left({ }^{28}\right)$ and also to ${ }^{8}$ for similar discussions on problems that are connected with thermodynamics for two-phase flow. 
e) Evolution of the liquid density $\rho_{l}$ :

If we assume that $C>0$, then:

$$
\rho_{l} \geq 0 \Longleftrightarrow \tau \geq \tau_{v}^{s}(P)(1-C)
$$

We set $\Psi_{5}=\tau-\tau_{v}^{s}(P)(1-C)$. The density $\rho_{l}$ and the function $\Psi_{5}$ have then the same sign. The governing equation on $\Psi_{5}$ is:

$$
\partial_{t}\left(\Psi_{5}\right)+U \partial_{x}\left(\Psi_{5}\right)+\left[(1-C) \hat{\gamma} P \frac{d}{d P} \tau_{v}^{s}(P)-\tau\right] \partial_{x}(U)=(1-C) \frac{\partial_{C}\left(e_{H R M}\right)}{\partial_{P}\left(e_{H R M}\right)} \Gamma \frac{d}{d P} \tau_{v}^{s}(P)+\Gamma \tau_{v}^{s}(P)
$$

As in subsection $d$ ) or $b$ ), this equation allows $\Psi_{5}$ to be positive or negative. Thus nothing ensures that $\rho_{l}$ remains positive.

\section{B. Entropy inequality}

We now account for viscous contribution, focusing on:

$$
\left\{\begin{array}{l}
\partial_{t}(\rho C)+\partial_{x}(\rho C U)=\rho \Gamma \\
\partial_{t}(\rho)+\partial_{x}(\rho U)=0 \\
\partial_{t}(\rho U)+\partial_{x}\left(\rho U^{2}+P\right)=\partial_{x}\left(\mu \partial_{x}(U)\right) \\
\partial_{t}\left(\rho E_{H R M}\right)+\partial_{x}\left(U\left(\rho E_{H R M}+P\right)\right)=\partial_{x}\left(\mu U \partial_{x}(U)\right)
\end{array}\right.
$$

together with $E_{H R M} \stackrel{\text { def }}{=} e_{H R M}(P, \rho, C)+\frac{U^{2}}{2}$. Restricting to regular solutions, and rewriting the latter system in terms of $(\rho, U, P, C)$, we get the following governing equations:

$$
\left\{\begin{array}{l}
\partial_{t}(\rho)+U \partial_{x}(\rho)+\rho \partial_{x}(U)=0 \\
\partial_{t}(U)+U \partial_{x}(U)+\frac{1}{\rho} \partial_{x}(P)=\frac{1}{\rho} \partial_{x}\left(\mu \partial_{x}(U)\right) \\
\partial_{t}(P)+U \partial_{x}(P)+\hat{\gamma}_{H R M} P \partial_{x}(U)=-\Gamma \frac{\partial_{C}\left(e_{H R M}\right)}{\partial_{P}\left(e_{H R M}\right)}+\frac{\mu}{\rho}\left(\partial_{x}(U)\right)^{2} \\
\partial_{t}(C)+U \partial_{x}(C)=\Gamma
\end{array}\right.
$$

Thus, introducing the entropy-entropy flux pair $\left(\eta_{H R M}, F_{\eta}^{H R M}\right)$, where $\eta_{H R M}=-\rho \ln \left(S_{H R M}\right)$ and $F_{\eta}^{H R M}=$ $-\rho U \ln \left(S_{H R M}\right)$, and assuming $S_{H R M}$ agrees with (20), we get:

$$
\partial_{t}\left(\eta_{H R M}\right)+\partial_{x}\left(F_{\eta}^{H R M}\right)=-\frac{\rho}{S} \partial_{P}\left(S_{H R M}\right)\left(\frac{\mu}{\rho}\left(\partial_{x}(U)\right)^{2}-\frac{\partial_{C}\left(e_{H R M}\right)}{\partial_{P}\left(e_{H R M}\right)} \Gamma\right)-\frac{\rho}{S} \partial_{C}\left(S_{H R M}\right) \Gamma
$$

and thus:

$$
\partial_{t}\left(\eta_{H R M}\right)+\partial_{x}\left(F_{\eta}^{H R M}\right)-\frac{\rho}{S} \partial_{P}\left(S_{H R M}\right) \Gamma\left(\frac{\partial_{C}\left(e_{H R M}\right)}{\partial_{P}\left(e_{H R M}\right)}-\frac{\partial_{C}\left(S_{H R M}\right)}{\partial_{P}\left(S_{H R M}\right)}\right)=-\frac{\rho}{S} \partial_{P}\left(S_{H R M}\right) \frac{\mu}{\rho}\left(\partial_{x}(U)\right)^{2} \leq 0
$$

Obviously, we retrieve the standard entropy inequality (21) when the mass transfer term $\Gamma$ is not accounted for. 


\section{References}

${ }^{1}$ A. Ambroso, C. Chalons, F. Coquel, E. Godlewski, F. Lagoutiere, P. A. Raviart and N. Seguin, Working group on the interfacial coupling of models, http://www.ann-jussieu.fr/groupes/cea, 2003.

2 , The coupling of homogeneous models for two-phase flows, Int. J. on Finite Volumes, http://www.latp.univmrs.fr/IJFV/, 2007, vol. 4, pp. 1-39.

3 _ Extension of interface coupling to general lagrangian systems, Math. of Comp., to appear.

${ }^{4}$ A. Ambroso, C. Chalons, F. Coquel, E. Godlewski, F. Lagoutiere and P.A. Raviart, Coupling of multiphase flow models, in the proceedings of NURETH 11, Avignon, France, 2-6 October, 2005.

${ }^{5} \mathrm{M}$. BAUdin, Méthodes de relaxation pour la simulation des écoulements diphasiques dans les conduites pétrolières, $\mathrm{PhD}$ thesis, Université Paris VI, France, 2003.

${ }^{6}$ M. Baudin, C. Berthon, F. Coquel, R. Masson and Q.H. Tran, A relaxation method for two-phase flow models with hydrodynamic closure law, Numerische Mathematik, 2005, vol. 99, pp. 411-440.

${ }^{7}$ T. Buffard, T. Gallouët and J.M. HÉrard, A sequel to a rough Godunov scheme. Application to real gases, Computers and Fluids, 2000, vol. 29-7, pp. 813-847.

${ }^{8} \mathrm{~F}$. Caro, Modélisation et simulation numériques des transitions de phase liquide-vapeur, $\mathrm{PhD}$ thesis, Ecole Polytechnique, France, Novembre 2004, in French.

${ }^{9}$ F. Caro, F. Coquel, D. Jamet and S. Kokh, A simple finite volume method for compressible isothermal two-phase flow simulation, Int. J. on Finite Volumes, http://www.latp.univ-mrs.fr, 2006.

${ }^{10}$ C. Chalons, P. A. Raviart and N. Seguin, The interface coupling of the gas dynamics equations, Quart. Appl. Math., to appear.

${ }^{11} \mathrm{~F}$. Coquel and B. Perthame, Relaxation of energy and approximate Riemann solvers for general pressure laws in fluid dynamics equations, SIAM J. Numer. Anal., 1998, vol. 35-6, pp. 2223-2249. In Memory of Ami Harten.

${ }^{12}$ R. Eymard, T. Gallouët and R. Herbin, Finite Volume Methods, In Handbook of Numerical Analysis (Vol. VII), editors : P.G. Ciarlet and J.L. Lions, pp. 729-1020, North-Holland, 2000

${ }^{13}$ T. Galié, A. Ambroso, C. Chalons and F. Coquel, Interface model coupling via prescribed local flux balance, 18th AIAA CFD Conference, AIAA paper 2007-3821, http://www.aiaa.org, 2007.

${ }^{14} \mathrm{~T}$. GallouËt, J.M. Hérard and N. Seguin, A hybrid scheme to compute contact discontinuities in one dimensional Euler systems, Math. Mod. and Num. Analysis, 2002, no36, pp. 1133-1159.

15 _ Some recent Finite Volume schemes to compute Euler equations using real gas EOS, Int. J. for Num. Meth. in Fluids, 2002, vol. 39, pp. 1073-1138.

16 _ Numerical modelling of two-phase flows using the two-fluid two-pressure approach, Math. Mod. Meth. in Appl. Sci., 2004, vol. 14, pp. 663-670.

${ }^{17}$ P. Goatin And P. G. Le Floch, The Riemann problem for a class of resonant nonlinear hyperbolic systems of balance laws, Ann. Inst. Henri Poincaré - Analyse Non Linéaire, 2004, vol. 21, pp. 881-902.

${ }^{18} \mathrm{E}$. Godlewski and P. A. Raviart, The numerical interface coupling of nonlinear hyperbolic systems of conservation laws: 1.the scalar case, Numerische Mathematik, 2004, vol. 97, pp. 81-130. 1996.

${ }^{19}$ E. Godlewski ANd P.A. RAviart, Numerical analysis for hyperbolic systems of conservation laws, Springer Verlag,

${ }^{20}$ E. Godlewski, K. C. Le Thanh and P. A. Raviart, The numerical interface coupling of nonlinear hyperbolic systems of conservation laws: 2.the case of systems, Math. Mod. Num. Anal., 2005, vol. 39, pp. 649-692.

${ }^{21}$ J.M. Greenberg And A.Y. Le Roux, A well balanced scheme for the numerical processing of source terms in hyperbolic equation, SIAM J. Numer. Anal., 1996, vol. 33-1, pp. 1-16.

${ }^{22}$ A. Guelfi and S. Рiтot, THYC (ThermoHYdraulique des Composants) Version 4.1 - Note de Principe, EDF-DRD Report (unpublished) HI-84-03-020A, 2004. In French.

${ }^{23}$ J.M. HÉrARD, A rough scheme to couple free and porous media, Int. J. on Finite Volumes, http://www.latp.univmrs.fr/IJFV/, 2006, vol. 3-2, pp. 1-28.

${ }^{24}$ J.M. HÉRARD AND O. HuRISSE, The numerical coupling of a two-fluid model with an homogeneous model, in preparation.

25 _ Boundary conditions for the coupling of two-phase flow models, 18th AIAA CFD Conference, AIAA paper 200\%4458, http://www.aiaa.org, 2007.

$26 \_$, Coupling two and one dimensional Euler equations through a thin interface, Computers and Fluids, 2007, pp. 651666.

${ }^{27}$ O. Hurisse, Couplage interfacial instationnaire de modèles diphasiques, PhD thesis, Université Aix Marseille I, France, 16/10/2006, in French.

${ }^{28}$ S. JAOUEN, Etude mathématique et numérique de stabilité pour des modèles hydrodynamiques avec transition de phase, PhD thesis, Université Paris VI, France, Novembre 2001, in French.

${ }^{29}$ R. Lewandowski and B. Mohammadi, Existence and positivity results for the phi-theta and a modified k-epsilon turbulence models, Math. Mod. and Meth. in Applied Sciences, 1992, vol. 3, pp. 195-215.

${ }^{30} \mathrm{P}$. RASCle AND O. MoRvant, Interface utilisateur de Thetis - THErmodynamique en Tables d'InterpolationS, EDF-DER Report HT-13/95021B, unpublished, 1995. In French. French.

${ }^{31}$ I. Toumi, FLICA IV : Manuel de référence. Méthode numérique, CEA Report (unpublished) DMT93/439, 1995. In 